"Lipid modified oligonucleotides conjugates: Insights into gene silencing, interaction with model membranes and cellular uptake mechanisms" Ugarte-Uribe, B., Grijalvo, S., Nuñez Pertiñez, S., Busto, J.V., Martín, C., Alagia, A., Goñi, F.M., Eritja, R., Alkorta, I. Bioorg. Med. Chem, 25(1), 175-186 (2017). doi: 10.1016/j.bmc.2016.10.024

\title{
Lipid-Modified Oligonucleotide Conjugates: Insights into Gene Silencing, Interaction with Model Membranes and Cellular Uptake Mechanisms
}

Begoña Ugarte-Uribe ${ }^{1}$, Santiago Grijalvo ${ }^{2,3}$, Samuel Núñez Pertíñez ${ }^{2}$, Jon V. Busto ${ }^{1}$, César Martín ${ }^{1}$, Adele Alagia ${ }^{2}$, Félix M. Goñi ${ }^{1}$, Ramón Eritja ${ }^{2,3}$, Itziar Alkorta ${ }^{1}$ *

${ }^{1}$ Instituto Biofisika (UPV/EHU, CSIC), and Departamento de Bioquímica, Universidad del País Vasco, P.O. 644, Bilbao 48080, Spain

2 Institute of Advanced Chemistry of Catalonia (IQAC-CSIC), Jordi Girona 18-26, E-08034 Barcelona, Spain

${ }^{3}$ Networking Center on Bioengineering, Biomaterials and Nanomedicine (CIBER-BBN)

* Correspondence: Email address: itzi.alkorta@ehu.es (I. Alkorta); Tel: +34-94-6012568; fax: $+34-94-6013500$

Keywords: Antisense technology, Cellular uptake, Gene delivery, Lipidoligonucleotide conjugates, Model membrane systems 


\begin{abstract}
The ability of oligonucleotides to silence specific genes or inhibit the biological activity of specific proteins has generated great interest in their use as research tools and therapeutic agents. Unfortunately, their biological applications meet the limitation of their poor cellular accessibility. Developing an appropriate delivery system for oligonucleotides is essential to achieve their efficient cellular uptake. In the present work a series of phosphorothioate lipid-oligonucleotide hybrids were synthesized introducing covalently single or double lipid tails at both 3'- and 5'-termini of an antisense oligonucleotide. Gene transfections in cultured cells showed antisense luciferase inhibition without the use of a transfecting agent for conjugates modified with the double-lipid tail at 5'-termini. The effect of the double lipid-tailed modification was further studied in detail in several model membrane systems as well as in cellular uptake experiments. During these studies the spontaneous formation of self-assembled microstructures is clearly observed. Lipidation allowed the efficient incorporation of the oligonucleotide in HeLa cells by a macropinocytosis mechanism without causing cytotoxicity in cells or altering the binding properties of the oligonucleotide conjugates. In addition, both single- and double-tailed compounds showed a similar behavior in lipid model membranes, making them useful in nucleotide-based technologies.
\end{abstract}




\section{Introduction}

Nucleic acid-based therapy is acknowledged as a promising approach to treat a large number of diseases. ${ }^{1}$ Antisense, small interfering RNA (siRNA), aptamers and exon-skipping are the most used mechanisms to generate novel drugs based on nucleic acids. For this reason, nucleic acid therapy has been considered as an alternative to small molecule drugs. ${ }^{2}$ However, serious drawbacks regarding stability and cellular uptake need to be still improved. The use of non-viral vectors in combination with nucleic acids has remarkably increased in the last decades due to its simple large scale production and low host immunogenicity. ${ }^{3}$

There are two major strategies for associating non-viral vectors with nucleic acids. The formulation approach is often one of the most commonly-used strategies since the combination between vector and cargo is carried out electrostatically. ${ }^{4}$ The second approach involves the use of a covalent strategy that allows the non-viral vector to be directly linked to nucleic acids. The latter strategy leads to stable conjugates that normally improve their biological properties in terms of stability, pharmacokinetic profiles and internalization without altering the properties of natural nucleic acids. ${ }^{5}$ This approach has allowed the development of synthetic strategies to obtain covalently polymers,${ }^{6}$ peptides, ${ }^{7}$ lipids,${ }^{8}$ among others ${ }^{9}$ conjugated with nucleic acids.

Lipids are commonly used for modifying oligonucleotides. ${ }^{10}$ The importance of such hybrid conjugates has been demonstrated in areas like nanobiotechnology, ${ }^{11}$ cell biology $^{12}$ and medicine. ${ }^{5}$ From a therapeutic point of view, lipid-oligonucleotide conjugates (LOCs) have emerged as an alternative to cationic particles in order to reduce toxicity and improve biodistribution. ${ }^{13}$ Nonetheless, a few studies deal with their biophysical and biochemical properties and their interactions with cellular 
components. ${ }^{14-17}$ Oligonucleotide entry into cells normally takes place through receptormediated endocytosis by binding with high affinity to cell surface receptors. ${ }^{18}$ However, this uptake efficiency could change when modified oligonucleotides are internalized into the cell.

In this context, a number of issues remain unsolved such as the LOCs interaction with the plasma membrane, the mechanism by which these kinds of conjugates are internalized and their final destination within cells. ${ }^{19}$ These pathways may vary with the membrane coat composition, the transport vesicle size and the fate of the internalized materials. Additionally, understanding of how cellular uptake and subcellular trafficking of lipid oligonucleotide conjugates is an essential starting point to gain insight into pharmacological effectiveness and gene silencing activities of LOCs. Many synthetic strategies have been reported in recent years for obtaining oligonucleotides covalently linked to a variety of lipophilic moieties..$^{20,21}$ The conjugation of these units can occur at either the nucleoside or the nucleotide level, giving rise to the corresponding amphiphilic-based compounds. ${ }^{20}$ Other strategies have incorporated lipid moieties through a phosphodiester bond in order to obtain LOC conjugates with better pharmacokinetic behaviors and improved cellular uptake. In general, the oligonucleotide ends (3'- and 5'-termini) are the most accessible conjugation sites for lipophilic units. $^{21}$

The primary aim of the current study is to synthesize a small library made up of phosphorothioate LOCs which contains a series of neutral lipids that were covalently introduced at both the 3'- and 5'-termini. This library was initially designed to evaluate the efficiency for knocking down the mRNA Renilla luciferase gene through antisense technology. We also evaluated its ability to provide suitable cellular uptake and inhibit luciferase gene expression in the absence of a transfection agent. After selecting the 
optimal LOC, we explored its physicochemical and biological properties in model membranes with different lipid compositions and its internalization into cells. Our results constitute a starting point for the development of novel lipid modifications and more effective amphiphilic oligonucleotides for antisense, aptamer, exon-skipping and single- or double-stranded siRNA technologies. ${ }^{22}$ 


\section{Materials and Methods}

All chemicals, reagents and solvents were purchased from Sigma-Aldrich (St Louis, MO) or Acros Organics (Geel, Belgium) and were used as received. All the standard phosphoramidites (A, C, G and T) and ancillary reagents used for oligonucleotide synthesis were purchased from Applied Biosystems (Foster City, CA) or Link Technologies (Glasgow, UK). Phosphorothioate oligonucleotide sequences: 5'-CGT TTC CTT TGT TCT GGA-3' (Luc, 19, Table 1) and 5'-CTG TCT GAC GTT CTT TGT-3' (Scr, Table 1) were purchased from Sigma-Aldrich (St Louis, MO). The sequences 5'-TCC AGA ACA AAG GAA ACG-3' (Table 1), 5'-CTC TCG CAC CCA TCT CTC TCC TTC T-3' (GEM91, Table 1) and 5'-A GAA GGA GAG AGA TGG GTG CGA GAG-3' (22, Table S1 in Supporting Information) were synthesized in house (phosphate form). Additional nuclease-free water was also prepared by using $0.1 \%(\mathrm{v} / \mathrm{v})$ of diethylpyrocarbonate (DEPC) to ensure the removal of RNase contamination. Luciferase assay kits were purchased from Promega (Fitchburg, WI). Qiagen Giga plasmid purification kit was purchased from Qiagen (Hilden, Germany). 1,2-dioleoyl-L- $\alpha$-phosphatidylcholine (DOPC), egg sphingomyelin (eSM) and cholesterol (Chol) were from Avanti Polar Lipids (Alabaster, AL). Mouse monoclonal antibody to mannose-6-phosphate receptor (M6PR), rabbit polyclonal antibody to clathrin heavy chain (HC) and rabbit polyclonal antibody to caveolin-1 were from Abcam (Cambridge, UK) and HRP-linked Goat anti-rabbit IgG antibody was supplied by Cell Signaling Technology (Danvers, MA). Lipofectamine 2000, Oligofectamine, OptiMEM, Alexa Fluor 546 phalloidin, Alexa Fluor 546 Donkey anti-mouse IgG (H+L) antibody, Alexa Fluor 633 Goat anti-rabbit $\operatorname{IgG}(\mathrm{H}+\mathrm{L})$ antibody, Hoechst 33258, DNA stain, DiD dye, SYBR® Gold nucleic acid gel stain and $1 \mathrm{~Kb}$ Plus DNA Ladder were from Life Technologies (Carlsbad, CA). Caveolin-1 siRNA, clathrin HC siRNA and 
rabbit polyclonal antibody to glyceraldehyde-3-phosphate dehydrogenase (GAPDH) were purchased from Santa Cruz Biotechnology (Santa Cruz, CA, USA). 5-( $N, N-$ Dimethyl)amiloride hydrochloride (DMA), chlorpromazine hydrochloride (CPZ), cytochalasin D (Cyt D), genistein, nocodazole, nystatin and the VenorGEM Mycoplasma Detection Kit were supplied from Sigma (St Louis, MO).

\subsection{Organic synthesis}

All reactions were carried out under an inert atmosphere of dry nitrogen. Reaction and mixture of compounds were monitored by TLC on silica gel (F254 Merck plates). TLC plates were visualized with phosphomolybdic acid in ethanol (20\%). Flash chromatography was performed using SDS 0.063-0.2 mm/70-230 mesh silica gel. ${ }^{1} \mathrm{H}$ and ${ }^{13} \mathrm{C}$ NMR spectra were recorded at $25^{\circ} \mathrm{C}$ on a Varian Mercury $400 \mathrm{MHz}$ spectrometer (Unitat de RMN, IQAC-CSIC) using deuterated solvents. Tetramethylsilane (TMS) was used as an internal reference $(0 \mathrm{ppm})$ for ${ }^{1} \mathrm{H}$ spectra and the residual signal of the solvent $(77.16 \mathrm{ppm})$ for ${ }^{13} \mathrm{C}$ spectra, both recorded in $\mathrm{CDCl}_{3}$. For $\mathrm{CD}_{3} \mathrm{OD}$ the residual signal of the solvent was used as a reference. Chemical shifts are reported in part per million (ppm) in the d scale, coupling constants $(J)$ in $\mathrm{Hz}$ and multiplicity as follows: $\mathrm{s}$ (singlet), $\mathrm{d}$ (doublet), $\mathrm{t}$ (triplet), $\mathrm{q}$ (quadruplet), quint (quintuplet), sext (sextuplet), m (multiplet) and br (broad signal). Electrospray ionization mass spectra (ESI-MS) were recorded on a Micromass ZQ instrument with single quadrupole detector coupled to an HPLC, and high-resolution (HR) ESI-MS on an Agilent 1100 LC/MS-TOF instrument (Servei d'Espectrometría de Masses, Universitat de Barcelona). Absorbance measurements and melting temperature studies were recorded on a Jasco FP-6200 spectrofluorometer equipped with a thermoregulated cell holder as well. 


\subsubsection{Synthesis of the double-tailed lipid derivatives 12 and 13}

Scheme 1 shows the main steps of the synthesis of the double-lipid derivatives. Further information regarding experimental procedures can be found in Supporting Information.

\subsubsection{Synthesis of the phosphorothioate LOCs}

Antisense LOCs (LOC-14, -15, -16, -17) (Table 1 and Supporting Information) were synthesized on a DNA/RNA synthesizer (Applied Biosystems) on a $1 \mu$ mol scale using the corresponding lipid functionalized CPG-solid supports (CPG-1, -2, -3) and the lipid phosphoramidite-4 (Figure 1). Coupling yields were $>97 \%$. The resulting DMTonoligonucleotides (LOC-14, -15, -16) and LOC-17 were deprotected and cleaved from the solid supports with aqueous concentrated ammonia $(32 \%)$ at $55^{\circ} \mathrm{C}$ overnight. The mixtures were filtered and ammonia solutions were concentrated to dryness. DMToncrudes and LOC-17 were purified by semi-preparative HPLC. Solvent A: 5\% ACN in $100 \mathrm{mM}$ triethylammonium acetate (TEAA) $(\mathrm{pH}=7)$ and solvent $\mathrm{B}: 70 \%$ ACN in 100 mM TEAA $(\mathrm{pH}=7)$. Column: $X$ XBidge $^{\mathrm{TM}}$ OST $\mathrm{C}_{18}$ semipreparative column $(10 \times 50$ $\mathrm{mm}, 2.5 \mathrm{~mm}$ ). Flow rate: $3 \mathrm{~mL} / \mathrm{min}$. Conditions: $15 \mathrm{~min}$ of linear gradient from 0 to $85 \%$ of B. The pure DMTon-peak was concentrated and dissolved in $\mathrm{AcOH}(80 \%)$ in order to remove the last DMT protecting group. After $30 \mathrm{~min}$, the deprotected antisense oligonucleotides were washed with ethylic ether and finally desalted with SephadexG25 (NAP-10 column). The isolated oligonucleotides LOC-14, -15, -16, -17 were finally analysed by HPLC (Column: XBridge ${ }^{\mathrm{TM}}$ OST $\mathrm{C}_{18}$ column; (4.6x50 mm, 2.5 $\mathrm{mm}$ ); Flow rate: $1 \mathrm{~mL} / \mathrm{min}$ ) and analysed by MALDI-TOF mass spectrometry (Table 1 and Supporting Information). 
The oligonucleotide conjugate $\mathrm{T}_{10} \mathbf{- 1 8}$ was isolated following the same experimental conditions described above (Table 1 and Supporting Information).

2.1.3 Synthesis of fluorescently labelled DNA sequence 24

For Alexa Fluor 488 labeling, a commercially available 5'-amino-modifier-C6-Tfa phosphoramidite (Glen Research) was introduced into the sequence 5'-A GAA GGA GAG AGA TGG GTG CGA GAG-3' (22, Table S1 and Supporting Information) obtaining the respective 5'-protected amino-derivative (23, Table S1 and Supporting Information). The CPG solid support was deprotected according to experimental conditions described above. Finally, oligonucleotide amino derivative $\mathbf{2 3}$ was analyzed by HPLC and confirmed by MALDI-TOF mass spectrometry (Table S1 and Supporting Information). 23 was used without further purification in the next step.

A solution of the deprotected oligonucleotide $\mathbf{2 3}$ was passed through a column filled with Dowex 50x2, sodium form to remove the interfering ammonium ions coming from the cleavage step. It was dissolved with $50 \mu \mathrm{L}$ of an aqueous solution of $0.2 \mathrm{M}$ $\mathrm{NaHCO}_{3}(\mathrm{pH}$ 9). Then, 1.1 equiv of Alexa Fluor 488 tetrafluorophenyl ester dissolved in $30 \mu \mathrm{L}$ DMF was added to the solution and left to react overnight at room temperature. The mixture was concentrated to dryness, and the residue was dissolved in $\mathrm{H}_{2} \mathrm{O}$ (1 mL). The green solution was desalted with Sephadex G-25 (NAP-10 Column) and the anticipated fluorescently labelled oligonucleotide 24 (Table S1 and Supporting Information) was purified by semi-preparative HPLC and confirmed by MALDI-TOF mass spectrometry.

\subsection{Thermal denaturation studies}

The thermal melting curves for duplexes formed by the phosphorothioate LOCs (LOC-14, -15, -16, -17), or the unmodified oligonucleotide 19, and the DNA 
complementary strand in its phosphate form (mixed in the appropriate buffer to a 1:1 molar ratio) were carried out recording the absorbance changes at $260 \mathrm{~nm}$ with a linear temperature ramp of $0.5^{\circ} \mathrm{C} / \mathrm{min}$ (Figure $\mathrm{S} 1$ in Supporting Information). The measurements were recorded on a JASCO V-650 spectrophotometer equipped with a Peltier temperature control. Sample concentrations were $1 \mu \mathrm{M}$ in $100 \mathrm{mM}$ phosphate buffer supplemented with $100 \mathrm{mM} \mathrm{NaCl}, \mathrm{pH}$ 7.02. Measurements were performed in triplicate.

\subsection{Formation of DNA duplexes}

Double-stranded LOC hybrids were prepared by mixing LOC-20 or LOC-21 with the sequence complementary strand $\mathbf{2 2}$ at equimolar ratio. For fluorescence experiments one of the duplex strands was labelled with Alexa 488. The annealing procedure was performed by incubating samples dissolved in phosphate-buffered saline (1× PBS) solution at $95^{\circ} \mathrm{C}$ for $2 \mathrm{~min}$, followed by a cooling step at $1^{\circ} \mathrm{C} / \mathrm{min}$ to room temperature in a temperature-controlled water circulating bath Julabo F10-MH (Julabo Labortechnik, Seelbach, Germany). The formation of the DNA duplexes was checked by either non-denaturing polyacrylamide or agarose gel electrophoresis (Figure S2 in Supporting Information). DNA duplexes were stored at $-20^{\circ} \mathrm{C}$.

\subsection{Transfection studies carried out with or without lipofectamine}

The luciferase inhibition assay was carried out on Mycoplasma free-HeLa (human cervical carcinoma) cell cultures that were maintained in exponential growth (Figure S3 in Supporting Information). Cells were seeded one day prior to the experiment in a 24well plate at a density of $10^{5}$ cells/well. Transfection of the antisense LOCs (LOC-14, - 
15, -16, -17) were carried out in the absence or presence of Lipofectamine 2000 as described by the manufacturer for adherent cell lines.

For Lipofectamine-treated cells, pRL-TK $(0.1 \mu \mathrm{g})$ and pGL3 $(1.0 \mu \mathrm{g})$ plasmids, which were used as a reporter and control, together with the phosphorothioate LOCs (LOC-14, -15, -16, -17) $(60 \mathrm{nM})$, were formulated into liposomes and added to each well with a final volume of $600 \mu \mathrm{L}$. After an incubation period of $20 \mathrm{~h}$, cells were washed once with PBS and were lysed with passive lysis according to the manufacturer's instructions.

To evaluate the ability of the LOC conjugates to silence mRNA Renilla luciferase, DMEM either supplemented with $10 \%$ FBS or without FBS, was used to carried out transfection experiments in the absence of Lipofectamine 2000. Firstly, pRL-TK (0.1 $\mu \mathrm{g})$ and pGL3 $(1.0 \mu \mathrm{g})$ plasmids were transfected by using commercially available cationic lipids. After $5 \mathrm{~h}$ incubation, cells were thoroughly washed with PBS $(2 \times 500$ $\mu \mathrm{L}$ ) and fed with fresh medium (either supplemented with $10 \%$ FBS, or without FBS, depending on the type of the transfection experiment). Phosphorothioate LOCs (LOC14, -15, -16, or -17) were added at a particular concentration and incubated for $24 \mathrm{~h}$. After $24 \mathrm{~h}$ cells were lysed with passive lysis buffer according to the instructions of the Dual-Luciferase Reporter Assay System. An unmodified antisense oligonucleotide 19 and a scramble oligonucleotide were used as controls. The luciferase activities of the samples were measured in a luminometer using the Luciferase Assay Reagent II and Stop and Glo Reagent. Lumminiscence was measured by using SpectraMax M5 spectrophotometer. The inhibitory effects generated were expressed as normalized ratios between the activities of the reporter (Renilla) luciferase gene and the control (firefly) luciferase gene. 


\subsection{Surface tension measurements}

The studies were carried out at $22^{\circ} \mathrm{C}$ using a $\mu$ Trough-S equipment (Kibron Inc., Finland) consisting of a small $(2-\mathrm{cm})$ rounded multi-well plate that allowed for $1 \mathrm{~mL}$ subphase measurements. For possible LOC-21 interaction with lipids, DOPC:eSM:Chol (2:2:1) monolayers were formed at the air-buffer interface at increasing initial pressures by spreading a small amount of lipid (about $2 \mathrm{nmol})$ in chloroform:methanol $(2: 1, \mathrm{v} / \mathrm{v})$ solution over $1 \times$ PBS. After allowing for solvent evaporation, LOCs were injected into the subphase with constant stirring and the changes in surface pressure were recorded. The plateau values of surface pressure increase $(\Delta \pi)$ after LOC insertion were plotted as a function of initial $\pi$ value $\left(\pi_{i}\right)$, and line fitted. Average values \pm SD $(n=2)$.

\subsection{SPB and GUV preparation}

SPB preparation was performed as described previously. ${ }^{23}$ Sucrose-enclosing GUVs were prepared using the electroformation method as described previously. ${ }^{23}$ DOPC, DOPC/eSM/Chol (2:2:1) and eSM:Chol (2:1) stock solutions were prepared containing $0.4 \mathrm{~mol} \%$ DiD (Invitrogen, Waltham, Massachusetts, USA). Vesicles were generated in a $300 \mathrm{mM}$ sucrose solution at $60^{\circ} \mathrm{C}$, a temperature rendering bilayers in a fluid phase state. $30 \mathrm{~min}$ after electroformation and vesicle equilibration at room temperature, $50 \mu \mathrm{L}$ vesicles were pipetted onto $250 \mu \mathrm{L}$ of an equiosmolar $1 \times$ PBS buffer solution in BSA-pre-treated Lab-Tek Chamber slides (Thermo Fisher Scientific, NY, USA).

\subsection{Analysis of LOC incorporation into GUVs and SPBs by confocal microscopy}

Images were obtained in an inverted fluorescence confocal microscope (Leica TCSSP5, Leica Microsystems, Wetzlar, Germany). Briefly, BSA-pre-treated 8 well- 
Lab-Tek Chamber slides containing GUVs or Biocell-liquid-cell containing SPBs were placed on the microscope followed by image acquisition with a $40 \times$ LD objective using an excitation wavelength of 488 for Alexa 488 and $633 \mathrm{~nm}$ for DiD, and the emission was recovered between 505 and $540 \mathrm{~nm}$ for Alexa 488 and 655 and $795 \mathrm{~nm}$ for DiD. Binding of Alexa 488-labelled LOC-21 to fluorescently labelled GUVs or SPBs was observed at $22^{\circ} \mathrm{C}$ under the microscope in sequential mode imaging. Image treatment was performed using Leica Application Suite software.

\subsection{Cellular binding/uptake studies by flow cytometry}

HeLa cells seeded in 24 -well plates $\left(1.25 \times 10^{5}\right.$ cell/well) were pretreated with endocytic inhibitors for $30 \mathrm{~min}$ at $37^{\circ} \mathrm{C}$ in OptiMEM followed by incubation with 500 nM Alexa 488-labeled LOC-21 for $2 \mathrm{~h}$ at $37^{\circ} \mathrm{C}$.Selected inhibitors: $0.3 \mathrm{M}$ sucrose, 10 $\mu \mathrm{g} / \mathrm{mL}$ CPZ, $5 \mu \mathrm{g} / \mathrm{mL}$ filipin, $200 \mu \mathrm{M}$ genistein, $100 \mu \mathrm{M}$ DMA, $10 \mu \mathrm{g} / \mathrm{mL}$ Cyt $\mathrm{D}, 20$ $\mu \mathrm{M}$ nocodazole were tested (Figure S4 in Supporting Information). After incubation, cells were completely dissociated with $0.5 \%$ Trypsin-EDTA, washed by centrifugation at $520 \times g$ for $10 \mathrm{~min}$ and resuspended into ice-cold $1 \times \mathrm{PBS}$, followed by a flow

cytometry analysis (FACSCalibur, Beckton Dickinson) analyzing $10^{4}$ cells per sample. The fluorescence of Alexa 488-labeled LOC-21 remaining at the cellular surface was also quenched with $0.2 \%$ Trypan Blue (final concentration) to measure only the fluorescence corresponding to the internalized LOC-21. Alexa 488 fluorescence was measured using an air-cooled $488 \mathrm{~nm}$ argon-ion laser, collecting emission signals by FL1 detector. GMFI (Geometric Mean Fluorescence Intensity) data of histograms were used for analysis comparison using WinMDI 2.9 and Cell Quest (BD Biosciences) softwares.

\section{9 siRNA-mediated silencing protein expression}


The expression of clathrin heavy chain and caveolin-1 proteins were silenced by siRNA treatment. siRNA transfection protocols were performed following the manufacturer's instructions. Cells seeded in 24 -well plates $\left(1.25 \times 10^{5}\right.$ cell/well $)$ were treated with siRNA-oligofectamine mixtures in OptiMEM. After siRNA incubation for 4-5 h, $1 \mathrm{~mL}$ opti-MEM containing 20\% FBS was added to the wells without removal of the transfection mixture. The cells were incubated further for $24 \mathrm{~h}$, the medium was replaced by appropriate growth medium, and cells were incubated for another $24 \mathrm{~h}$. Cells were scrapped, counted and used for cellular uptake assays and Western blotting experiments in order to test siRNA silencing efficiency.

\subsection{Western blotting}

After siRNA treatment, HeLa cells were harvested and lysed in ice-cold homogenization buffer. Proteins were electrophoretically separated on a $12 \%$ SDSpolyacrylamide gel and transferred to nitrocellulose membranes. The membranes were then blocked for $1 \mathrm{~h}$ with 5\% skim milk in Tris-buffered saline (TBS) containing $0.01 \%$ $\mathrm{NaN}_{3}$ and $0.1 \%$ Tween 20 , then incubated overnight with the primary antibody $(1: 1,000$ dilution) in TBS- $0.1 \%$ Tween 20 at $4^{\circ} \mathrm{C}$. After three washes with TBS-0.1\% Tween 20 , membranes were incubated with horseradish peroxidase-conjugated secondary antibody at 1:4,000 dilution for $1 \mathrm{~h}$. Equal loading of protein was monitored using a specific antibody against GAPDH protein. Proteins were detected using the enhanced chemiluminiscence detection system (ECL®, Amersham Biosciences). The Quantity

One® Image Analyzer software program (Bio-Rad, Hercules, CA) was used for quantitative densitometric analysis.

\subsection{Cellular localization assays by confocal microscopy}


Cells seeded in Poly-L-lysine pre-treated 8 well-Lab-Tek Chamber slides $\left(5 \times 10^{4}\right.$ cell/well) were pre-incubated with endocytic inhibitors (100 $\mu \mathrm{M}$ DMA, 0.3 M sucrose or $5 \mu \mathrm{g} / \mathrm{mL}$ filipin) for $2 \mathrm{~h}$ at $37^{\circ} \mathrm{C}$, followed by incubation with $100 \mathrm{nM}$ Alexa 488 labeled LOC-21 for $30 \mathrm{~min}$ at $37^{\circ} \mathrm{C}$ in OptiMEM. After incubation, cells were washed three times, fixed with $4 \%(\mathrm{w} / \mathrm{v})$ paraformaldehyde for $15 \mathrm{~min}$ at room temperature (RT) and permeabilized with $0.1 \%(\mathrm{w} / \mathrm{v})$ Triton X-100 dissolved in $1 \times$ PBS for $10 \mathrm{~min}$ at RT. Next, samples were blocked with $1 \%(\mathrm{w} / \mathrm{v})$ BSA dissolved in $1 \times$ PBS at RT for 30 min. After blocking samples were incubated with the appropriate primary antibodies (1:200 dilution) at RT for $1 \mathrm{~h}$ followed by incubation with the appropriate Alexaconjugated secondary antibodies (1:200 dilution) at RT for $1 \mathrm{~h}$. Then samples were stained with nuclear marker Hoechst 33258 at RT for 10 min and visualized using Olympus IX 81 inverted fluorescence confocal microscope. Image acquisition was performed with a digital camera (Axiocam NRc5, Zeiss). Samples were placed on top of the microscope followed by image acquisition with a $60 \mathrm{X}$ oil immersion objective, using an excitation wavelength of 405, 488, 543 and $633 \mathrm{~nm}$ for Hoechst 33258, Alexa 488, Alexa 546 and Alexa 633, respectively. The emission was recovered between 430$460 \mathrm{~nm}$ for Hoechst 33258, 505-525 nm for Alexa 488, 560-600 nm for Alexa 546, 660IF nm for Alexa 633. Image treatment was performed using Fluoview v.50 software (Olympus Life Science, Pennsylvania, USA).

\subsection{Statistical analysis}

All measurements were performed at least 3 times, and results are presented as mean \pm s.d. Levels of significance were determined by a two-tailed Student's $t$-test, and a confidence level $(* p<0.05, * * p<0.01, * * * p<0.001)$ was used to establish statistical significance. 


\section{Results and discussion}

\subsection{Synthesis of phosphorothioate lipid-oligonucleotide conjugates}

The strategy followed for introducing lipophilic residues at the 3'- and 5'-termini of an antisense oligonucleotide was based on obtaining the appropriate modified CPG-solid supports $^{21}$ (CPG-1, -2, -3) and the lipid phosphoramidite-4, ${ }^{21}$ respectively (Figure 1). Following this strategy, small series of siRNA conjugates containing neutral and cationic residues were obtained and their RNAi silencing activities were previously evaluated in cell culture. We found that a modified siRNA conjugate containing a lipid double-tailed pendant group at the 5'-termini showed the best transfection efficiency compared to other lipophilic conjugates also synthesized. ${ }^{21}$ Inspired by these results, the development of a synthetic strategy to introduce the same double lipid-tailed modification at the 3'-termini of a phosphorothioate oligonucleotide was found particularly interesting.

The synthesis of the solid support 3 (CPG-3) containing the double lipid-tailed modification with two aliphatic chains of fourteen carbon atoms in length is depicted in Scheme 1. First, the double alkylation of our starting material $\mathbf{5}$ in the presence of a phase-transfer catalyst and $50 \% \mathrm{NaOH}$ solution in toluene gave the corresponding alkylated compound $\mathbf{6}$ in $52 \%$ yield. ${ }^{24}$ Next, Boc-deprotection in acid conditions $(30 \%$ trifluoroacetic acid in dichloromethane) produced the anticipated amine in its trifluoroacetate form that was converted to the anticipated free amine derivative 7 by using a cationic ion exchange resin in carbonate form. ${ }^{25}$

INSERT FIGURE 1. Lipid functionalized CPG-solid supports (CPG-1, CPG-2, CPG-3) used for the synthesis of antisense LOCs at the 3'-termini (A) and the lipid-phosphoramidite derivative 
(phosphoramidite-4) used for modifying the 5'-termini of an antisense phosphorothioate (PS) and DNA phosphate (PO) oligonucleotides (B).

We then studied both dimethoxytrityl and trityl as protecting groups to selectively block the primary alcohol of the triol 8. This reaction generated the anticipated protected diols $\mathbf{9}$ and $\mathbf{1 0}$ in 40 and $70 \%$ yield, respectively. In order to combine the amino lipid $\mathbf{7}$ and the diols $\mathbf{9}$ and $\mathbf{1 0},{ }^{26,27}$ we thought about obtaining the matching carbamate as the best approach for getting the modified lipid derivative with a trityl residue. Although the introduction of $p$-nitrophenyl chloroformate into the amino lipid 7 worked well in our hands and quantitatively yielded the carbamate 11, several attempts to displace the leaving group with the appropriate alcoxide (generated from the diols 9 or $\mathbf{1 0}$ in the presence of sodium hydride) were not as efficient as we originally thought they would be. The anticipated lipophilic compounds $\mathbf{1 2}$ and $\mathbf{1 3}$ were achieved although none of the reactions exceeded a $15 \%$ yield. Finally, with the tritylated lipid derivative 13 in our hands, we were able to functionalize CPG solid support according with wellestablished methods ${ }^{28}$ yielding the expected modified solid support CPG-3 $(L=44$ $\mu \mathrm{mol} / \mathrm{gr})$.

INSERT SCHEME 1. Synthesis of the Controlled Pore Glass (CPG)-solid support 3 containing the double-tailed lipid modification used for the conjugation of an antisense oligonucleotide at 3'-termini. (a) $\mathrm{C}_{14} \mathrm{H}_{29} \mathrm{Br}, \mathrm{Bu}_{4} \mathrm{NHSO}_{4}, 50 \% \mathrm{NaOH}$, Toluene, $60^{\circ} \mathrm{C}$, overnight; (b) i. $30 \%$ TFA in $\mathrm{CH}_{2} \mathrm{Cl}_{2}, \mathrm{RT}, 1 \mathrm{~h}$; ii. Carbonate resin, AcOEt, RT, 1 h; (c) 4-nitrophenyl chloroformate, $\mathrm{CH}_{2} \mathrm{Cl}_{2}$ :THF (1:1), DIPEA, RT, 3 h; (d) $\mathrm{NaH}(60 \%), 9$ or 10, DIPEA, DMF, overnight at RT or $60^{\circ} \mathrm{C}$, for 9 and 10, respectively; (e) i. 13, succinic anhydride, DMAP, $\mathrm{CH}_{2} \mathrm{Cl}_{2}$, RT, overnight; ii. CPG functionalization; (f) DMTr-Cl or Tr-Cl, DMAP, pyridine, $6 \mathrm{~h}$ at $\mathrm{RT}$ or $60^{\circ} \mathrm{C}$ for $\mathrm{DMTr}-\mathrm{Cl}$ and $\mathrm{Tr}-\mathrm{Cl}$, respectively.

Two sequences modified with lipids (Luc and GEM91, Table 1) were selected for the present work. The first one corresponds to a 18-mer phosphorothioate oligonucleotide (Luc: 5'-CGT TTC CTT TGT TCT GGA-3') that is complementary to the coding 
region of the mRNA Renilla luciferase gene. ${ }^{29}$ The second one corresponds to a phosphate 25-mer oligonucleotide (GEM91: 5'-CTC TCG CAC CCA TCT CTC TCC TTC T-3') which is complementary to the gag initiation site of HIV. ${ }^{30}$ The covalent introduction of neutral lipid residues into sequence 19 (Table 1) at 3'-termini was carried out from the CPG-solid supports (CPG-1, -2, -3). In addition, the lipid phosphoramidite- $4^{21}$ was also incorporated at the 5 '-termini of the same antisense oligonucleotide. Once the sequences were completed, detachment from the solid support and full deprotection of the nucleobases protecting groups were achieved using an overnight treatment with ammonia $(32 \%)$ at $55^{\circ} \mathrm{C}$ (see Materials and Methods section for further experimental details). Purification of the antisense LOCs (LOC-14, -15 and 16) was carried out according to DMTon-based protocols, except for the LOC-17 DMTr protecting group, which was deprotected at the end of the DNA synthesis). All LOCs were analyzed by HPLC and finally characterized by MALDI-TOF mass spectrometry (Table 1 and Supporting Information), affording the expected MS except for LOC-16 which gave a mass that corresponded to the loss of the double lipid moiety. To confirm this result, we coupled the same solid-support CPG-3 on a decathymidine sequence at the 3'-termini which afforded the conjugate $\mathrm{T}_{10}$-18. Although the MS analysis by MALDI-TOF showed the same loss of the lipid residue observed earlier, ESI experiments confirmed the exact mass of the conjugate $\mathrm{T}_{10} \mathbf{- 1 8}$ (Table 1 and Supporting Information).

The effect of the terminal lipids at the 3'- and 5'-end on the hybridization properties of the LOC conjugates (LOC-14, -15, -16, -17) was measured by thermal denaturation analysis of the corresponding duplexes. Results are displayed on Table 1 and Figure S1 (Supporting Information). These data showed similar thermal stabilities in all cases $\left(50.3,51.5,51.5\right.$ and $50.9^{\circ} \mathrm{C}$ for LOC-14, $\mathbf{- 1 5}, \mathbf{- 1 6}$ and $\mathbf{- 1 7}$, respectively) with respect to 
the unmodified sequence $19\left(50.8^{\circ} \mathrm{C}\right)$. These results indicated that introducing lipid pendants at the ends of a phosphorothioate oligonucleotide single strand did not cause any significant destabilizing effect on the duplex structure. Similar results were reported in literature when hydrocarbonated lipids were introduced at the end of the siRNA passenger strand. ${ }^{21,31,32}$

INSERT TABLE 1. MALDI-TOF mass spectrometry and melting temperatures for lipidoligonucleotide conjugates shown in this work

\subsection{In vitro gene silencing studies}

The silencing activity of the amphiphilic phosphorothioate LOCs (LOC-14, -15, 16, -17) and the effect of the lipophilic moieties in antisense technology were studied in mammalian cells using the unmodified oligonucleotide (19) as a control. First, the silencing efficiency of the lipophilic LOCs on Renilla mRNA knockdown was evaluated in serum-free conditions at $60 \mathrm{nM}$ in the absence of a transfecting agent in order to avoid unwanted side effects of FBS on the transfection process (Figure 2A). All amphiphilic conjugates were able to silence luciferase activity $(46 \%, 23 \%, 26 \%$ and 49\% for LOC-14, LOC-15, LOC-16 and LOC-17, respectively). The highest antisense silencing efficiencies were shown by LOC-14 and LOC-17 (46\% and 49\%, respectively) which improved the silencing activity compared to the unmodified phosphorothioate $19(22 \%)(* p<0.01$ and $* * * p<0.001$ for LOC-14 and LOC-17, respectively). The silencing efficiency for LOC-15 and LOC-16 showed that the presence of the alkenyl and the saturated double-lipid residues at the 3'-termini was not as effective as LOC-14 and LOC-17 (23 and 26\%, versus 46 and 49\%, respectively).

Although it has been described that both the lipophilicity degree and the position of the hydrocarbonated chain may affect the cellular uptake process, ${ }^{33}$ we did not expect 
big differences between LOC-16 and LOC-17. A potential source for these differences $(* * * p<0.001)$ could be hypothesized due to differences on their degradability capabilities. Thus, in the case of LOC-17, the 5'-phosphate hydrolysis between the double-lipid alkyl chain at the 5'-end and the antisense oligonucleotide might keep intact the antisense oligonucleotide and might facilitate endosomal escape enhancement. Once released, it would be ready to recognize and block a particular mRNA. As a result, it would improve the corresponding silencing efficiency.

Unexpectedly, this luciferase inhibition efficiency was not found in the case of LOC-16 as well as for other 3'-LOCs, in our hands. This difference might be due to the presence of threoninol-based lipid modification at the 3'-termini which might slow down the hydrolysis process. Thus, the oligonucleotide-3'-lipid would remain longer in the endosomal compartment. Interestingly, during our studies on model membranes explained below, we observed that the oligonucleotide-lipid carrying the double lipid at the 5'-end (LOC-21) had a tendency to self-assemble into larger structures and these process may facilitate cellular uptake process. The linker needed to attach the double linker at the 3'-end may disrupt this self-assembling process, disturbing this phenomenon. Moreover, we found that LOC-21 showed and enhanced internalization via receptor-mediated endocytosis in $\beta 2$ integrin (CR3) expressing cells ${ }^{23}$ but nothing is known for oligonucleotide-3'-lipid. Hence, this clearly indicates that the modification at the $5^{\prime}$-end enables the binding and interaction between the oligonucleotide and its interacting partners, such as $\beta 2$ integrin receptor.

LOC-14 and LOC-17, which afforded the best results in Figure 2A, were selected for further transfection studies. We evaluated the LOCs silencing activities in the presence of a commercially available transfecting agent and 10\% FBS at $60 \mathrm{nM}$ of LOC in order to study whether the effect of lipid modifications together with their position in 
the oligonucleotide (3'- or 5'-termini) could disrupt the antisense machinery (Figure 2B). We also included LOC-16 because was modified with the same double lipid residue than LOC-17 but at the 3'-termini. Although all conjugates inhibited luciferase expression $(61 \%, 46 \%$ and $10 \%$ for LOC-14, LOC-16 and LOC-17, respectively), these inhibition results were lower than the inhibition showed by the unmodified phosphorothioate oligonucleotide 19, which displayed the best inhibition profile (82\%) of the series. Specifically, the silencing activities diminished gradually when lipid modifications containing one or two hydrocarbon branches were introduced at the 3'termini (61\% and 46\% for LOC-14 and LOC-16, respectively) $(* p<0.05)$. Regarding the hydrocarbon chain position (either 3'- or 5'-termini), the inhibition activity of Renilla luciferase in the presence of lipofectamine was practically abolished in our hands when the double lipid modification was introduced at the 5'-termini (10\% versus $61 \%$ and $46 \%$ for LOC-17, LOC-14 and LOC-16, respectively). Interestingly, a significant reduction of luciferase activity in the case of LOC-14 was achieved when compared to the double-lipid modification introduced at the 3 '-termini $\left({ }^{*} p<0.05\right)$. If only compared the effect induced by the double-lipid tail, we found statistically differences between LOC-16 and LOC-17, respectively $(* * * p<0.001)$.

Based on these preliminary inhibition results, it is suggested that the kind of lipid modification (saturated versus unsaturated with one or two branches) together with their position in the antisense oligonucleotide tract (3'- or 5'- termini) could consider two important parameters in gene silencing process. Furthermore, the low effectiveness observed for the conjugate LOC-17 might point out not only to the unpacking of the LOC-17/lipofectamine lipoplexes but also to the inefficient interaction of LOC-17 with its interacting partners. This suggests that this modification could somehow disrupt the binding properties of the oligonucleotide. 
INSERT FIGURE 2. Analysis of Renilla mRNA knockdown in HeLa cells with different phosphorothioate LOCs (LOC-14, -15, 16, -17). (A) Normalized luciferase activity in serum-free conditions with a LOCs concentration in cell culture of $60 \mathrm{nM}$. (B) Normalized luciferase activity with phosphorothioate LOCs (LOC-14, -16 and -17) in the presence of lipofectamine at $60 \mathrm{nM}$. (C) Doseresponse experiment using phosphorothioate LOC-14 and LOC-17 at several concentrations (60, 150 and $300 \mathrm{nM}$ ). A phosphorothioate scramble (Scr) (without any modification) showed the specificity of the experiment at $60 \mathrm{nM}$. Phosphorothioate 19 (without any modification) was used as a positive control. Results are expressed as mean \pm SD for an average of three to six independent replicates. A regular twoway ANOVA variance analysis combined with Bonferroni post-test for multiple comparisons was used. $* * * \mathrm{p}<0.001, * * p<0.01, * p<0.05$ when the comparison was established between conditions indicated in $(\mathrm{A}),(\mathrm{B})$ or $(\mathrm{C})$ to establish statistical significance.

In an attempt to emulate the physiological conditions, we further investigated the ability of the phosphorothioate LOC-14 and LOC-17 to impart cellular uptake at several concentrations $(60,150$ and $300 \mathrm{nM})$ in the absence of lipofectamine but in the presence of serum proteins. As shown in Figure 2C, the antisense silencing activity of LOC-14 was drastically reduced in the presence of $10 \% \mathrm{FBS}(v / v)$, indicating that LOC-14 was not able to silence Renilla luciferase gene at the three concentrations tested. Conversely, LOC-17 could inhibit the Renilla luciferase expression in a dose-response manner. While antisense activity for LOC-17 was barely detected at 60 and $150 \mathrm{nM}$, luciferase expression was inhibited to $22 \%$ when a higher concentration was tested $(300 \mathrm{nM})(* p$ $<0.05)$. Similarly, this result improved the silencing activities achieved for the unmodified oligonucleotide 19 and LOC-14 at the same concentration (300 nM; ${ }^{*} p<$ 0.01). In view of these results, we could hypothesize, among other factors, that transfection efficiencies mediated by LOC-14 and -17 might be compromised due to the dependence and/or association of these two amphiphilic conjugates with serum proteins. $^{33}$ 
In order to understand the effect induced by this double-lipid modification, we characterized in detail the insertion of a DNA sequence modified with the same double lipid-tail at 5'-termini into model membranes (giant unilamellar vesicles, GUVs, and supported planar bilayers, SPBs) as well as its mechanism of entry in cultured cells.

\subsection{Insertion into model membranes}

We selected the GEM91 (gene-expression modulator 91) sequence (5'-CTC TCG CAC CCA TCT CTC TCC TTC T $\underline{X}-3^{\prime}$; where $X=3$-amino modifier C7-CPG) as a model DNA sequence to incorporate the lipid phosphoramidite-4 at the 5'-termini (LOC-20) since GEM91 oligonucleotide has been shown to be a potent inhibitor of HIV-1 replication in cell culture by interacting with the conserved gag initiation site of HIV $-1 .^{30}$ Furthermore, to carry out membrane binding studies and cellular uptake experiments, fluorescent dye Alexa Fluor 488 was introduced into the LOC-20 model conjugate at 3'-termini (LOC-21) taking advantage of the presence of the free primary amine group, as previously described. ${ }^{23}$ Although model membranes cannot mimic the complex cellular environment, their use would allow the study of certain biophysical aspects of oligonucleotide delivery under controlled experimental conditions and welldefined lipid compositions. ${ }^{34,35}$

We could clearly observe that this LOC could be efficiently inserted into DOPC/eSM/Chol (2:2:1) monolayers at initial $\pi$ above $35 \mathrm{mN} / \mathrm{m}$ (Figure S5A in Supporting Information), indicating that its incorporation into different domains of cell membranes would be expected since biological membranes are considered to support a lateral pressure $\pi \approx 30 \mathrm{mN} / \mathrm{m}$, as previously observed in several cell lines, ${ }^{23}$ albeit with large fluctuations around this average value. ${ }^{36}$ These results were in agreement with what was previously observed in GUVs and SPBs composed of the same ternary 
mixture, ${ }^{23}$ where the insertion of LOC showed a homogeneous incorporation into both liquid-disordered $\left(\mathrm{L}_{\mathrm{d}}\right)$ and liquid-ordered $\left(\mathrm{L}_{\mathrm{o}}\right)$ domains. In fact, this LOC showed first a rapid interaction with the $\mathrm{L}_{\mathrm{d}}$ phase of the bilayer, followed by its accumulation in the boundary between the $\mathrm{L}_{\mathrm{d}}$ and $\mathrm{L}_{\mathrm{o}}$ domains and finally leading to its incorporation to $\mathrm{L}_{\mathrm{o}}$ domains and the formation of Alexa 488-enriched rounded structures (Figure S5B in Supporting Information).

Next, GUVs and SPBs model membranes presenting pure $\mathrm{L}_{\mathrm{d}}$ (DOPC) and $\mathrm{L}_{\mathrm{o}}$ [eSM/Chol (2:1)] phase states were also used in order to study LOC-21 insertion into each pure phase and to analyze the possible dependence of the $\mathrm{L}_{0}-\mathrm{L}_{\mathrm{d}}$ interface on the insertion. Here, a rapid and remarkable interaction was observed with both model membranes, independently of the lipid composition, which also lead to the formation of Alexa 488-enriched structures in all SPBs tested (Figure S6 in Supporting Information). These findings helped us discard a possible influence of domain boundaries on the LOC insertion, suggesting that this LOC could interact and insert into either $\mathrm{L}_{\mathrm{d}}$ or $\mathrm{L}_{\mathrm{o}}$ phases within a cell membrane.

Finally, we investigated whether the bilayer-binding properties of this LOC in model membranes in a DNA duplex based conjugate. Two duplexes were formed to carry out this study: LOC-21 was hybridized with its complementary strand (22) in order to form a double-stranded hybrid that contained an Alexa 488 fluorophore in the 3'-termini (dsLOC-1) or LOC-20 was hybridized with the fluorescently labelled complementary sequence $\mathbf{2 4}$ bearing the Alexa 488 dye at 5'-termini of the complementary strand (dsLOC-2) (Figure S2 in Supporting Information). Oligonucleotide 24 covalently labelled with Alexa 488 was obtained from the 5'-aminooligonucleotide $\mathbf{2 3}$ following the experimental procedures described (see Materials and methods for further information). Interestingly, both double-stranded LOCs (dsLOC-1 
and dsLOC-2) behave similarly to LOC-21. They could easily insert into both $\mathrm{L}_{\mathrm{d}}$ and $\mathrm{L}_{\mathrm{o}}$ domains of GUVs and composed of DOPC, DOPC/eSM/Chol (2:2:1) and eSM/Chol (2:1) (Figure 3A). In addition, Alexa 488-enriched intense spots (bigger than $1 \mu \mathrm{m}$ in diameter) were also observed in SPBs composed of DOPC/eSM/Chol (2:2:1), suggesting that these reproducible microstructures are at least in part formed by these compounds (Figure 3B).

INSERT FIGURE 3. Double-stranded LOC binding to lipid bilayers. (A) Confocal microscopy images of DiD-labelled DOPC (upper panels), DOPC/eSM/Chol (2:2:1) (middle panels) and eSM/Chol (2:1) (bottom panels) GUVs (equatorial sections) incubated for $2 \mathrm{~h}$ with $100 \mathrm{nM}$ (50 nM Alexa 488labelled) double-stranded LOCs, carrying Alexa 488 fluorophore bound to the 3 '-end of the LOC (dsLOC-1, right panels) or to the 5'-end of the complementary strand (dsLOC-2, left panels). (B) Confocal microscopy images of DiD-labelled DOPC/eSM/Chol (2:2:1) SPBs incubated with $100 \mathrm{nM}$ dsLOC-1 (right panels) and dsLOC-2 (left panels). White squares represent the zoom taken, corresponding to the images below. Scale bars, $10 \mu \mathrm{m}$.

A deeper analysis of these reproducible Alexa 488-enriched structures was further performed by varying the zeta plane of the fluorescence confocal microscope. As seen in Figure 4, some of these bright structures were partially static in time (Figure 4A, arrowheads) whereas other dynamic structures were in continuous motion (Figure 4A, arrows). In addition, the dynamic structures seemed to be more tubular-like than rounded (Figure 4A, middle and lower panels), and they remained anchored to the bilayer along the time of the experiment. When a single Alexa 488-enriched rounded structure was analyzed, it was confirmed that this particular structure was localized at the boundary between $\mathrm{L}_{d}$ and $\mathrm{L}_{\mathrm{o}}$ phases (Figure $4 \mathrm{~B}$ ). Interestingly, when some of the static rounded structures were highly zoomed and laser-radiated, they became tubularshaped and dynamic, possibly due to photolytic structure destabilization. 
Overall, we can conclude that the increase in the hydrophilicity of the LOC by hybridization with its complementary strand does not seem to alter the binding properties of the lipid moiety. Hence, this lipid moiety could be considered a good conjugation anchor for a wide range of nucleic acid-based molecules, including singleand double-stranded compounds. In addition, this compound shows no lateral segregation when incorporated to bilayers in either its single- or double-form, thereby enhancing its chances to be internalized through several uptake pathways. In fact, LOCdriven generation of such microstructures might reinforce LOC preference for highly curved membranes connected with various internalization routes.

INSERT FIGURE 4. Alexa 488-enriched structures formed with dsLOC-1 in DiD-labelled DOPC/eSM/Chol (2:2:1) SPBs. (A) Imaging of Alexa 488-enriched structures from a single SPB zone. Arrowhead and arrow stand for more and less stable Alexa 488-enriched structures, respectively. (B) Imaging of a single Alexa 488-enriched structure. In both cases different z planes were taken from a single SPB zone. Scale bars, $10 \mu \mathrm{m}$.

\subsection{Internalization studies}

It was previously reported by our group that the internalization of LOC-21 was enhanced in $\beta 2$ integrin (CR3) expressing cells via receptor-mediated endocytosis. ${ }^{24}$ However, it still remains unclear how LOC-21 is internalized in cells lacking $\beta 2$ integrin receptor at the cell surface. In order to gain more insight into the mechanism of entry of this compound, flow cytometry and confocal microscopy studies were performed in mycoplasma free HeLa cells in the absence of serum in order to reduce unwanted interactions (Figure S3 in Supporting Information).

Since oligonucleotides covalently conjugated with lipids may be considered to be membrane permeable, as previously observed in both membrane lipid model systems and HeLa cells, ${ }^{23}$ we first performed cellular uptake experiments at different 
temperatures $\left(37^{\circ} \mathrm{C}\right.$ and $\left.4^{\circ} \mathrm{C}\right)$ in these cells to elucidate whether enhanced cellular uptake of these compounds was energy-dependent. Interestingly, internalization of the fluorescently labelled LOC-21 was significantly decreased at $4^{\circ} \mathrm{C}$ in comparison with that at $37^{\circ} \mathrm{C}$ (Figure S7 in Supporting Information). This observation may suggest that LOC uptake occurs most likely through energy-dependent endocytic pathways. ${ }^{37}$ Similar results were obtained by Nelson and co-workers who reported a temperaturedependent cellular uptake of myristoylated cargos. ${ }^{38}$

Then HeLa cells were treated with non-cytotoxic concentrations of several inhibitors of different endocytic routes prior to incubation with LOC-21, in order to investigate the role of a possible endocytosis mechanism (Figure 5; Figures S4 and S8 in Supporting Information). Pre-treatment of HeLa cells with filipin or genistein, both reported to block caveolae-mediated uptake processes, ${ }^{39,40}$ had no remarkable effect on its uptake. In contrast, cells pre-treated with $\mathrm{CPZ}$ or sucrose, which are known to perturb clathrin-mediated endocytosis, ${ }^{41,42}$ showed a more noticeably decreased of LOC-21 internalization, suggesting that this entry route might be involved in the LOC uptake (Figure 5A-B). Furthermore, the combination of sucrose and filipin did not cause any further decrease in the LOC uptake, suggesting that this inhibition could only be attributable to the effect of sucrose (Figure 5B). Pre-treatment with nocodazole showed similar results to those obtained by CPZ pretreatment (Figure 5A-B) which reinforced the view that microtubules could be in part involved in LOC internalization by interfering with the late phase of endocytosis from early to late endosomes. ${ }^{43,44}$

INSERT FIGURE 5. LOC-21 internalization pathway in HeLa cells. (A) Representative histograms and (B) flow cytometry values (Geometric Mean of Fluorescence Intensity, GMFI) of the cellular uptake of Alexa 488-labelled LOC-21 in HeLa cells in the presence of endocytic inhibitors. Results are expressed as GMFI of 5 independent experiments performed in duplicate (n.s. for filipin; $\mathrm{p}<0.05$ for CPZ and genistein; $p<0.01$ for the rest). (C) Representative Western blotting assay of clathrin HC and 
caveolin-1 protein expression in HeLa cells after treatment with the appropriate siRNA molecule. Bottom graph shows the results of scanning densitometry of the exposed film. Data are expressed as arbitrary units of intensity relative to control value (Control) which stands for untreated sample, results are the mean \pm SD of 2 independent experiments. (D) Cellular uptake of Alexa 488-labelled LOC-21 in HeLa cells pretreated with clathrin HC and caveolin-1 siRNAs. Clathrin HC siRNA and caveolin-1 siRNA stand for HeLa cells pretreated with clathrin HC siRNA and caveolin-1 siRNA, respectively. Results are expressed as GMFI of 3 independent experiments performed in duplicate ( $p<0.05$ for clathrin HC siRNA; $\mathrm{p}<0.01$ for caveolin-1 siRNA).

Cyt D and DMA were used to inhibit macropinocytosis, a process of bulk fluidphase endocytosis. ${ }^{45,46}$ Interestingly, by selecting this entry pathway this molecule could efficiently reach the nucleus and cytoplasm, avoiding the destabilizing lysosomal environment. The latter pre-treatments also diminished LOC uptake suggesting that macropinocytosis may also be involved in the LOC internalization (Figure 5A-B). In addition, siRNA treatment efficiently silenced the expression of clathrin $\mathrm{HC}$ and caveolin-1 proteins (Figure 5C-D). However, no remarkable decrease on LOC internalization was observed under these experimental conditions. In this case, higher internalization values were also obtained for the positive control. This increase in fluorescence could derive from the presence of less number of cells during LOC-21 incubation due to siRNA pre-treatment (i.e., a number of cells died or detached during siRNA pre-treatment). A possible explanation for the non-changed fluorescent values could be the compensation of the uptake process by other pathways such as macropinocytosis due to the inhibition of clathrin-mediated endocytosis. Similarly, the effect achieved by sucrose inhibitor was non-specific since it not only blocked clathrindependent endocytosis but also fluid-phase endocytosis, thereby affecting other endocytic pathways, such as micropinocytosis. ${ }^{47-49}$ Also, the incorporation of this LOC into both $\mathrm{L}_{\mathrm{d}}$ and $\mathrm{L}_{\mathrm{o}}$ domains could probably lead to an indirect low-degree internalization of the LOC by other entry pathways owing to its presence at the plasma membrane. 
In order to validate the above findings obtained in the presence of endocytic blockers, the cellular uptake and intracellular trafficking of LOC-21 were studied by fluorescence confocal microscopy, which could reveal the co-localization of several fluorescently-labelled cellular markers (Figure 6). In DMA-pre-treated cells, LOC-21 was mainly visualized at the plasma membrane, co-localizing with actin filaments (Figure 6, upper panels). Similarly, in the presence of hypertonic sucrose medium, poor LOC-21 internalization was observed with a remarkable co-localization of LOC and clathrin $\mathrm{HC}$ near the plasma membrane (Figure 6, middle panels). On the contrary, in filipin-pretreated cells, intracellular LOC fluorescence was easily visualized with a slight co-localization of M6PR, a late endosome marker (Figure 6, lower panels). ${ }^{50}$ According to these results, it appears that energy-dependent LOC internalization could be mediated by a macropinocytosis and clathrin-mediated endocytosis suggesting that microtubules may also be involved in the latter process.

INSERT FIGURE 6. Visualization of LOC-21 internalization in HeLa cells with endocytic markers in the presence of endocytic inhibitors. Cells pre-treated with DMA (A), sucrose (B) or filipin (C) were incubated with LOC-21 (green) prior to fixation. (A) Alexa Fluor 546-Phalloidin was used for actin staining (red). (B) Primary antibody to Clathrin HC and Alexa Fluor 633 secondary antibody were used for clathrin staining (magenta). (C) Primary antibody to M6PR and Alexa Fluor 546 secondary antibody were used for late endosome staining (red). In all cases Hoechst 33258 was used for nucleus staining (blue). White boxes stand for zoomed areas.

\section{Conclusions}

A number of lipid moieties formed by saturated and unsaturated alkyl chains were covalently introduced at the 3' and 5'-termini of an antisense oligonucleotide. Because of the interest in modifying oligonucleotides with lipids in order to increase their ability to cross cell membranes, a new synthetic strategy for introducing a double lipid-tailed moiety at the 3'-termini of an oligonucleotide (LOC-16) was described. LOC-16 was 
part of a small library based on several phosphorothioate oligonucleotides conjugated with a series of lipids either at 5'- or 3'-termini. The antisense silencing activity and the ability to impart cellular uptake of these phosphorothioate LOCs were evaluated in cell culture. The results indicated that the double lipid-tail introduced at 5'-termini (LOC20) was able to silence and reduce moderately luciferase levels. Additionally, we were able to characterize the binding of this double lipid-tailed modification into artificial membranes like GUVs and SPBs, where spontaneous self-assembled microstructures were clearly observed. Both single- and double-forms of this compound showed similar binding behavior, implying that the increase in the hydrophilicity of the LOC by hybridization with its complementary strand does not seem to alter the binding properties of the lipid moiety. Finally, macropinocytosis and clathrin-mediated endocytosis are selected as cellular entry mechanisms by this LOC. The LOC did not cause cytotoxicity or alter the binding properties of the oligonucleotide itself, thereby enabling binding to different molecules, such as intracellular proteins involved in the intracellular oligonucleotide transport, complementary strands and target mRNA molecules. The above results indicate that the double lipid-tailed oligonucleotide is a good starting point for both single- and double-stranded oligonucleotide delivery.

Supplementary Materials: Synthesis of the double-tailed lipid derivatives 12 and 13; functionalization of Controlled Pore Glass (CPG)-solid support; ${ }^{1} \mathrm{H}-\mathrm{NMR}$ and ${ }^{13} \mathrm{C}-\mathrm{NMR}$ spectra; HPLC and MALDI-TOF mass spectrometry chromatograms of both intermediates and final products; melting temperature studies, polyacrylamide and agarose gels of dsLOCs, mycoplasma test, cellular uptake of LOC-21 under different temperatures and cell cytotoxicity assay in the presence of endocytic inhibitors. 
Acknowledgments: This work is supported by the European Commission (Grants FP7NMP-213382-2, FUNMOL and NMP4-LA-2011-262943, MULTIFUN), by the Spanish Ministry of Education (Grant CTQ2014-52588-R, CTQ2014-61758-EXP and BFU2012-36241), the Generalitat de Catalunya (2009/SGR/208) and the Instituto de Salud Carlos III (CB06_01_0019). CIBER-BBN is an initiative funded by the VI National R\&D\&i Plan 2008-2011, Iniciativa Ingenio 2010, Consolider Program, CIBER Actions and financed by the Instituto de Salud Carlos III with assistance from the European Regional Development Fund.

Conflicts of Interest: The authors declare no conflict of interest.

\section{References and notes}

1. Li, T.; Wu, M.; Zhu, Y. Y.; Chen, J.; Chen, L. Nucleic Acid Ther. 2014, $24,302$.

2. Zhou, J.; Shum, K. T.; Burnett, J. C.; Rossi, J. J. Pharmaceuticals 2013, 6, 85.

3. $\quad$ Elsabahy, M.; Nazarali, A.; Foldvari, M. Curr. Drug Delivery 2011, 8, 235.

4. $\quad$ Allen, T. M.; Cullis, P. R. Adv. Drug Deliv. Rev. 2013, 65, 36.

5. Juliano, R. L.; Ming, X.; Nakagawa, O. Acc. Chem. Res. 2012, 45, 1067.

6. Loczenski, R. V.; Winkler, G. S.; Allen, S.; Puri, S.; Mantovani, G. Eur. Polym. J. 2013, 49, 2861.

7. Fabani, M. M.; Ivanova, G. D.; Gait, M. J. Therapeutic Oligonucleotides. Kurreck, J., editor. Cambridge, UK: Royal Society of Chemistry; 2008, pp 80102. 8. $\quad$ Raouane, M.; Desmaële, D.; Urbinati, G, Massaad-Massade, L.; Couvreur, P. Bioconjug. Chem. 2012, 23, 1091. 
9. Jeong, J. H.; Mok, H.; Oh, Y. K.; Park, T. G. Bioconjug. Chem. 2009, 20, 5.

10. Patwa, A.; Gissot, A.; Bestel, I.; Barthélémy, P. Chem. Soc. Rev. 2011, 40, 5844.

11. Werz, E.; Korneev, S.; Montilla-Martínez, M.; Wagner, R.; Hemmler, R.;

Walter, C.; Eisfeld, J.; Gall, K.; Rosemeyer, H. Chem. Biodivers. 2012, 9, 272.

12. Kubo, T.; Yanagihara, K.; Takei, Y.; Mihara, K.; Sato, Y.; Seyama, T. Mol.

Pharm. 2012, 9, 1374.

13. Raouane, M.; Desmaele, D.; Gilbert-Sirieix, M.; Gueutin, C.; Zouhiri, F.;

Bourgaux, C.; Lepeltier, E.; Gref, R.; Bensalah, R.; Clayman, G.; MassaadMassade, L.; Couvreur, P. J. Med. Chem. 2010, 54, 4067.

14. Teixeira, H.; Rosilio, V.; Laigle, A.; Lepault, J.; Erk, I.; Scherman, D.;

Benita, S.; Couvreur, P.; Dubernet, C. Biophys. Chem. 2001, 92, 169.

15. Gordon, S. P.; Berezhna, S.; Scherfeld, D.; Kahya, N.; Schwille, P. Biophys. J. 2005, 88, 305.

16. Bunge, A.; Kurz, A.; Windeck, A. K.; Korte, T.; Flasche, W.; Liebscher, J.; Hermann, A.; Huster, D. Langmuir 2007, 23, 4455.

17. Bunge, A.; Loew, M.; Pescador, P.; Arbuzova, A.; Brodersen, N.; Kang, J.;

Dahne, L.; Liebscher, J.; Herrmann, A.; Stengel, G.; Huster, D. J. Phys. Chem. B 2009, 113, 16425 .

18. Ding, Y.; Wang, W.; Feng, M.; Wang, Y.; Zhou, J.; Jianping, D.; Ding, X.; Zhou, X.; Liu, C.; Wang, R.; Zhang, Q. Biomaterials 2012, 33, 8893.

19. Watson, P.; Jones, A. T.; Stephens, D. J. Adv. Drug Deliv. Rev. 2005, 57, 43.

20. Gissot, A.; Camplo, M.; Grinstaff, M. W.; Barthélémy, P. Org. Biomol. Chem. 2008, 6, 1324. 
21. Grijalvo, S.; OCampo S. M.; Perales, J. C.; Eritja, R. Chem. Biodivers. 2010, $8,287$.

22. Elsner, M. Nature Biotech. 2012, 30, 1063.

23. Ugarte-Uribe, B.; Grijalvo, S.; Busto, J. V.; Martín, C.; Eritja, R.; Goñi, F. M.; Alkorta, I. Biochim. Biophys. Acta: General Subjects 2013, 1830, 4872.

24. Kokotos, G.; Verger, R.; Chiou, A. Chemistry Eur. J. 2000, 6, 4211.

25. Heyes, J.; Palmer, L.; Bremmer, K.; Bremmer, K.; MacLachlan, I. J. Control. Release 2005, 107, 276.

26. Oliviero, G.; Borbone, N.; Amato, J.; D’Errico, S.; Galeone, A.; Piccialli, G.; Varra, M.; Mayol, L. Biopolymers 2009, 91, 466.

27. Malla Reddy, S.; Venkat Reddy, Y.; Venkateswarlu, Y. Tetrahedron Lett. 2005, 46, 7439 .

28. Gupta, K. C.; Kumar, P.; Bhatia, D.; Sharma, A. K. Nucleosides and Nucleotides 1995, 14, 829.

29. Zhang, H. Y.; Mao, J.; Zhou, D.; Xu, Y.; Thonberg, H.; Liang, Z.; Wahlestedt, C. Nucleic Acid Res. 2003, 31, e72.

30. Turner, J. J.; Fabani, M.; Arzumanov, A. A.; Ivanova, G.; Gait, M. J. Biochim. Biophys. Acta. 2006, 1758, 290.

31. Shea, R. G.; Marsters, J. C.; Bischofberger, N. Nucleic Acids Res. 1990, 18, 3777.

32. Godeau, G.; Staedel, C.; Barthélémy, P. J. Med. Chem. 2008, 51, 4374.

33. Felber, A. E.; Bayó-Puxan, N.; Deleavey, G. F.; Castagner, B.; Damha, M. J.; Leroux, J-C. Biomaterials 2012, 33, 5955.

34. Werz, E.; Rosemeyer, H. Beilstein J. Org. Chem. 2015, 11, 913-929.

35. Werz, E.; Rosemeyer, H. Beilstein J. Org. Chem. 2014, 10, 2307-2321. 
36. Busto, J. V.; Sot, J.; Goñi, F. M.; Mollinedo, F.; Alonso, A. Biochim. Biophys. Acta 2007, 1768, 1855.

37. Bawa, R.; Fung, S. Y.; Shiozaki, A.; Yang, H.; Zheng, G.; Keshavjee, S.; Liu, M. Nanomed. Nanotech. Med. 2012, 8, 647.

38. Nelson, A. R.; Borland, L.; Allbritton, N. L.; Sims, C. E. Biochemistry 2007, 46, 14771.

39. Schnitzer, J. E.; Oh, P.; Pinney, E.; Allard, J. J. Cell Biol. 1994, 127, 1217.

40. Orlandi, P. A.; Fishman, P. H. J. Cell Biol. 1998, 141, 905.

41. Zhang, X.; Jin, Y.; Plummer, M. R.; Pooyan, S.; Gunaseelan, S.; Sinko, P. J. Mol. Pharm. 2009, 6, 836.

42. Wang, L. H.; Rothberg, K. G.; Anderson, R. G. J. Cell Biol. 1993, 123, 1107.

43. Zelphati, O.; Szoka, F. C. Proc. Natl. Acad. Sci. USA. 1996, 13, 11493.

44. Vasquez, R. J.; Howell, B.; Yvon, A. M.; Wadsworth, P.; Cassimeris, L. Mol. Biol. Cell. 1997, 8, 973.

45. Jackman, M. R.; Shurety, W.; Ellis, J. A.; Luzio, J. P. J. Cell Sci. 1994, 107, 2547.

46. Koivusalo, M.; Welch, C.; Hayashi, H.; Scott, C. C.; Kim, M.; Alexander, R. T. J. Cell Biol. 2010, 188, 547.

47. Bradley, J. R.; Johnson, D. R.; Pober, J. S. J. Immun. 1993, 12, 5544.

48. Synnes, M.; Prydz, K.; Lovdal, T.; Brech, A.; Berg, T. Biochim. Biophys. Acta 1999, $1421,317$.

49. Ivanov, A. I. Meth. Mol. Bio. 2008, 440, v.

50. Goldenberg, N. M.; Grinstein, S.; Silverman, M. Mol. Biol. Cell 2007, 18, 4762. 
FIGURE 1. Lipid functionalized CPG-solid supports (CPG-1, CPG-2, CPG-3) used for the synthesis of antisense LOCs at the 3'-termini (A) and the lipid-phosphoramidite derivative (phosphoramidite-4) used for modifying the 5'-termini of an antisense phosphorothioate (PS) and DNA phosphate (PO) oligonucleotides (B).
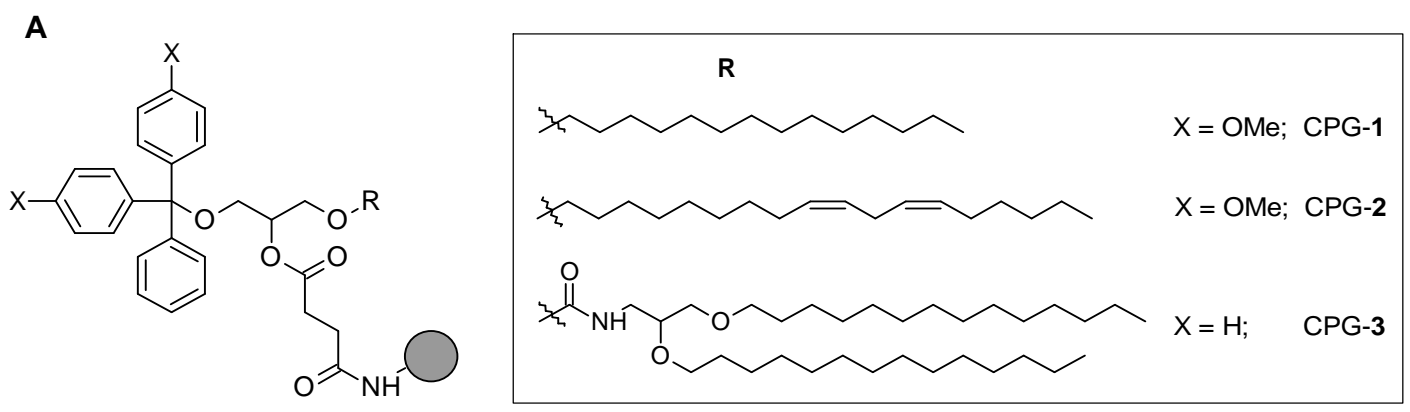

B

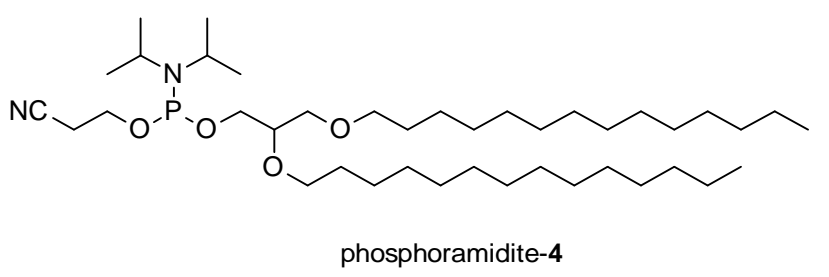


SCHEME 1. Synthesis of the Controlled Pore Glass (CPG)-solid support 3 containing the doubletailed lipid modification used for the conjugation of an antisense oligonucleotide at 3'-termini. (a) $\mathrm{C}_{14} \mathrm{H}_{29} \mathrm{Br}, \mathrm{Bu}_{4} \mathrm{NHSO}_{4}, 50 \% \mathrm{NaOH}$, Toluene, $60^{\circ} \mathrm{C}$, overnight; (b) i. $30 \%$ TFA in $\mathrm{CH}_{2} \mathrm{Cl}_{2}, \mathrm{RT}, 1 \mathrm{~h}$; ii. Carbonate resin, AcOEt, RT, 1 h; (c) 4-nitrophenyl chloroformate, $\mathrm{CH}_{2} \mathrm{Cl}_{2}$ :THF (1:1), DIPEA, RT, $3 \mathrm{~h}$; (d) $\mathrm{NaH}(60 \%), 9$ or 10, DIPEA, DMF, overnight at RT or $60^{\circ} \mathrm{C}$, for 9 and 10 , respectively; (e) i. 13, succinic anhydride, DMAP, $\mathrm{CH}_{2} \mathrm{Cl}_{2}$, RT, overnight; ii. CPG functionalization; (f) DMTr-Cl or $\mathrm{Tr}-\mathrm{Cl}$, DMAP, pyridine, $6 \mathrm{~h}$ at $\mathrm{RT}$ or $60^{\circ} \mathrm{C}$ for DMTr-Cl and $\mathrm{Tr}-\mathrm{Cl}$, respectively.
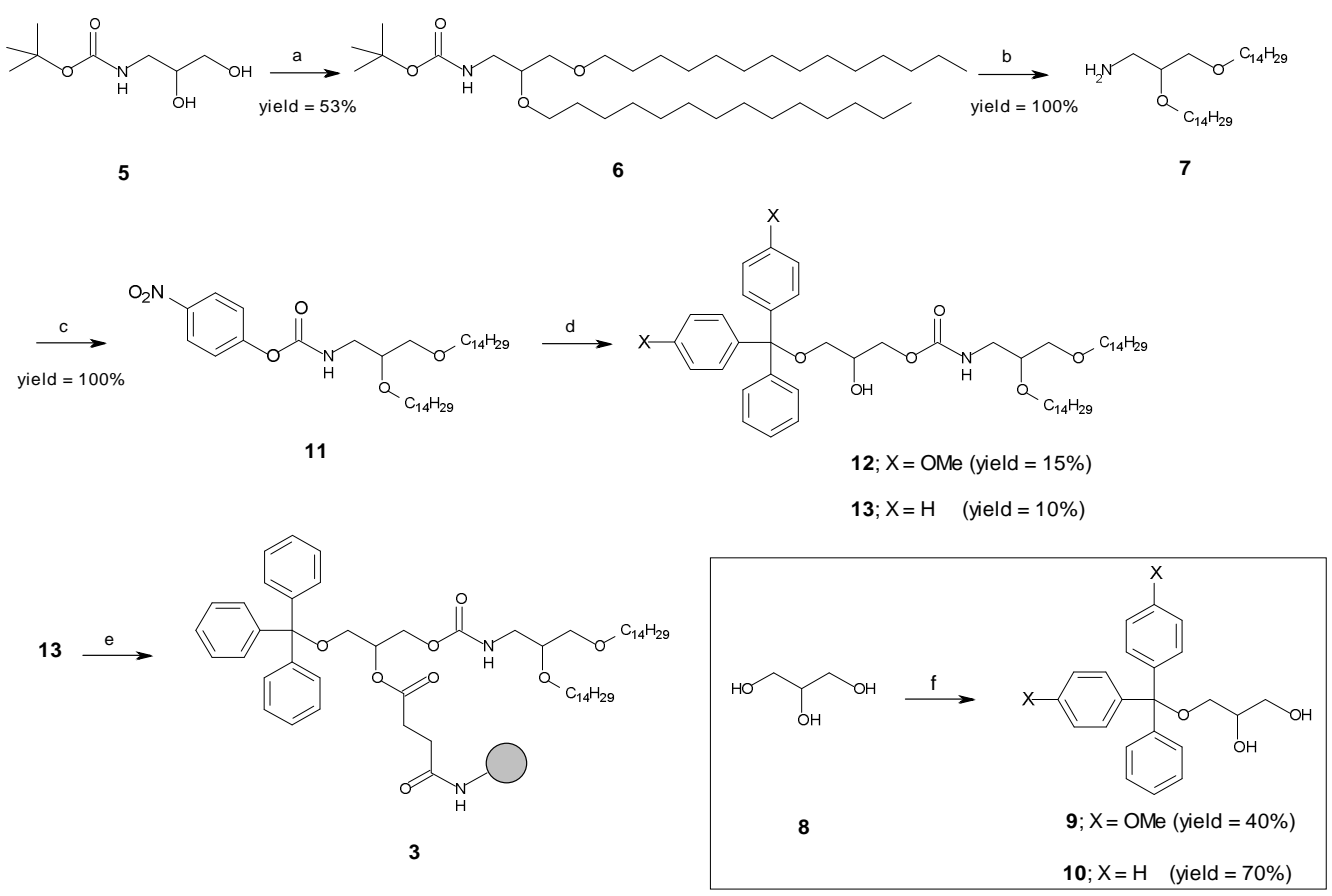
TABLE 1. MALDI-TOF mass spectrometry and melting temperatures for lipid-oligonucleotide conjugates shown in this work

\begin{tabular}{|c|c|c|c|c|c|c|}
\hline Name $^{1}$ & Modification $^{2}$ & Backbone $^{3}$ & $\begin{array}{c}\text { Lipid } \\
\begin{array}{c}\text { Oligonucleotide } \\
\text { Conjugate } \\
(\text { LOC })\end{array} \\
\end{array}$ & $\begin{array}{l}\text { Mass } \\
\text { [calcd] }\end{array}$ & $\begin{array}{l}\text { Mass } \\
\text { [found] }\end{array}$ & $\mathbf{T}_{\mathrm{m}} /{ }^{\circ} \mathrm{C}\left(\Delta \mathbf{T}_{\mathrm{m}}\right)^{4}$ \\
\hline Luc & $3^{\prime}-\mathrm{C}_{14}$ (CPG-1) & PS & LOC-14 & 6097 & 6095 & $50.3(-0.6)$ \\
\hline Luc & $3^{\prime}-\mathrm{C}_{18}$ (CPG-2) & PS & LOC-15 & 6131 & 6136 & $51.5(0.6)$ \\
\hline Luc & $3^{\prime}-\mathrm{C}_{28}$ (CPG-3) & PS & LOC-16 & 6410 & $6012^{7}$ & $51.5(0.6)$ \\
\hline Luc & $\begin{array}{c}5^{\prime}-\mathrm{C}_{28} \\
\text { (phosphoramidite-4) }\end{array}$ & PS & LOC-17 & 6276 & 6279 & $50.9(0.1)$ \\
\hline $\mathrm{T}_{10}$ & $3^{\prime}-\mathrm{C}_{28}(\mathrm{CPG}-3)$ & $\mathrm{PO}$ & $\mathrm{T}_{10^{-}}-18^{5}$ & 3643 & 3643 & nd \\
\hline Luc & unmodified & PS & 19 & nd & $\mathrm{Nd}$ & 50.8 \\
\hline Scr & unmodified & PS & Scr & nd & $\mathrm{Nd}$ & nd \\
\hline GEM91 & $\begin{array}{c}5 '-\mathrm{C}_{28} \\
\text { (phosphoramidite-4) }\end{array}$ & $\mathrm{PO}$ & LOC- $20^{6}$ & 8147 & 8149 & nd \\
\hline GEM91 & $\begin{array}{c}5^{\prime}-\mathrm{C}_{28} \\
\text { (phosphoramidite-4) }\end{array}$ & $\mathrm{PO}$ & LOC- $21^{6}$ & 8629 & 8631 & nd \\
\hline
\end{tabular}

${ }^{1}$ Luciferase (Luc): 5'-CGT TTC CTT TGT TCT GGA-3'; T $_{10}$ : Decathymidine; Scramble (Scr): 5'-CTG TCT GAC GTT CTT TGT-3'; GEM91: 5'-CTC TCG CAC CCA TCT CTC TCC TTC T-3'

${ }^{2} 3$ '-lipid at the 3'-termini or 5'-lipid at the 5'-termini and reagents used for the incorporation of the modification

${ }^{3}$ PS: phosphorothioate; PO: phosphodiester

${ }^{4} \Delta T_{m}$ corresponds to the difference between the melting temperatures of the corresponding phosphorothioate LOCs/antisense strand (phosphate form) hybrids minus the melting temperature of the unmodified phosphorothioate oligonucleotide (19)/antisense strand duplex (phosphate form; 5'-TCC AGA ACA AAG GAA ACG-3')

${ }^{5}$ see Supplementary Information for additional information: MALDI-TOFF mass spectrometry and HPLC chromatogram

${ }^{6}$ for more information see ref. 43

${ }^{7}$ main mass peak corresponds to the loss of the two $-\mathrm{C}_{14}$ aliphatic chains

nd: not determined 
FIGURE 2. Analysis of Renilla mRNA knockdown in HeLa cells with different phosphorothioate LOCs (LOC-14, -15, 16, -17). (A) Normalized luciferase activity in serum-free conditions with a LOCs concentration in cell culture of $60 \mathrm{nM}$. (B) Normalized luciferase activity with phosphorothioate LOCs (LOC-14, -16 and -17) in the presence of lipofectamine at $60 \mathrm{nM}$. (C) Doseresponse experiment using phosphorothioate LOC-14 and LOC-17 at several concentrations (60, 150 and $300 \mathrm{nM}$ ). A phosphorothioate scramble (Scr) (without any modification) showed the specificity of the experiment at $60 \mathrm{nM}$. Phosphorothioate 19 (without any modification) was used as a positive control. Results are expressed as mean \pm SD for an average of three to six independent replicates. A regular two-way ANOVA variance analysis combined with Bonferroni post-test for multiple comparisons was used. $* * * p<0.001, * * p<0.01, * p<0.05$ when the comparison was established between conditions indicated in (A), (B) or (C) to establish statistical significance.

A

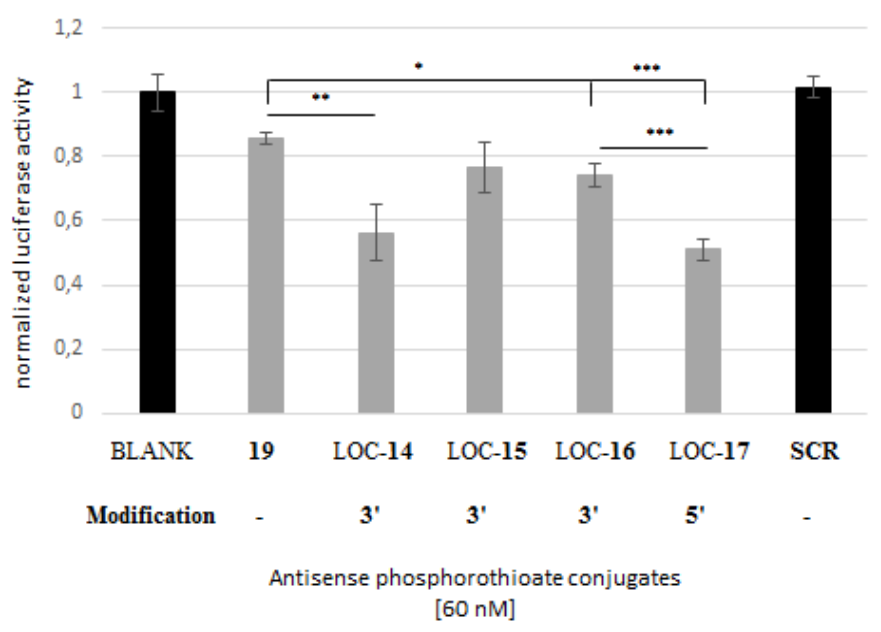




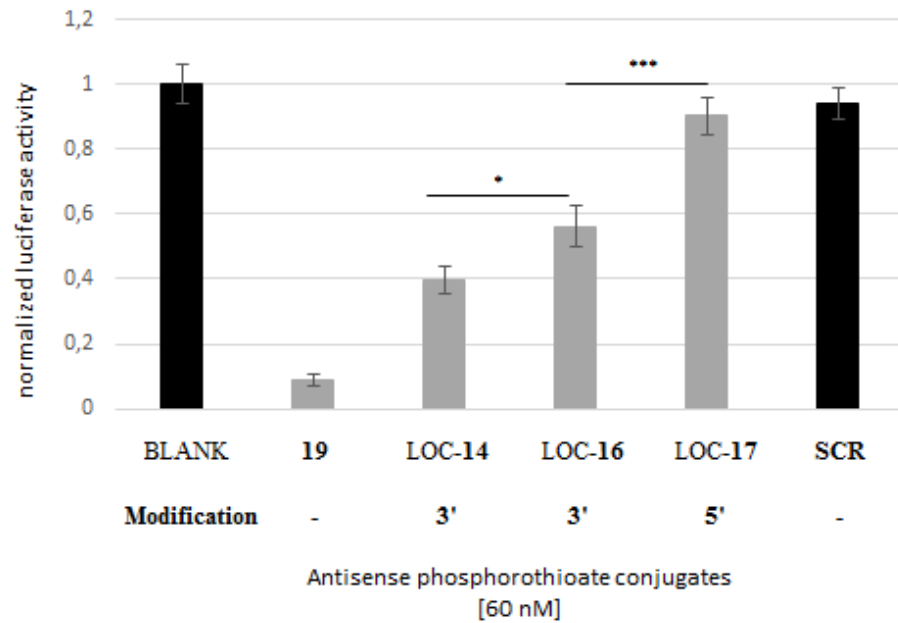

C

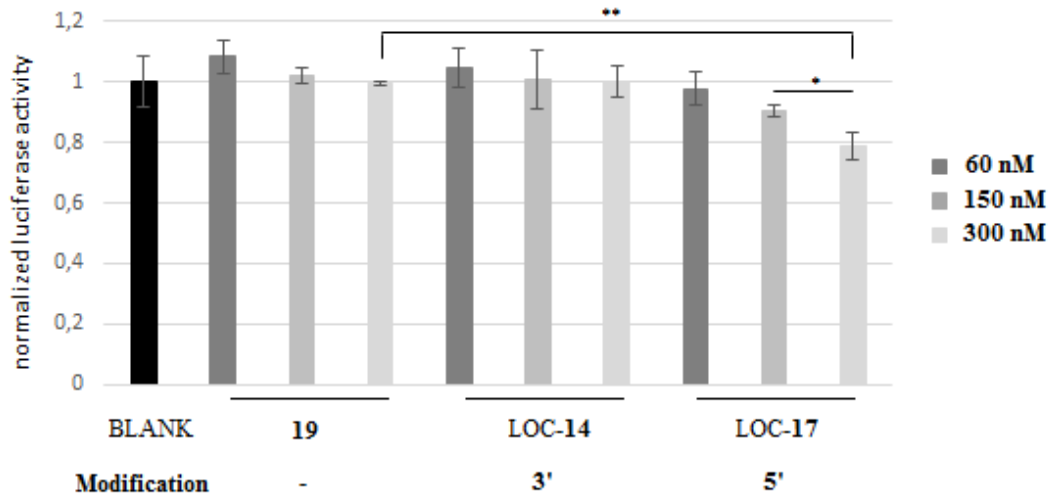

Antisense phosphorothioate conjugates 
FIGURE 3. Double-stranded LOC binding to lipid bilayers. (A) Confocal microscopy images of DiD-labelled DOPC (upper panels), DOPC/eSM/Chol (2:2:1) (middle panels) and eSM/Chol (2:1) (bottom panels) GUVs (equatorial sections) incubated for $2 \mathrm{~h}$ with $100 \mathrm{nM}$ (50 nM Alexa 488labelled) double-stranded LOCs, carrying Alexa 488 fluorophore bound to the 3'-end of the LOC (dsLOC-1, right panels) or to the 5'-end of the complementary strand (dsLOC-2, left panels). (B) Confocal microscopy images of DiD-labelled DOPC/eSM/Chol (2:2:1) SPBs incubated with 100 nM dsLOC-1 (right panels) and dsLOC-2 (left panels). White squares represent the zoom taken, corresponding to the images below. Scale bars, $10 \mu \mathrm{m}$.

A
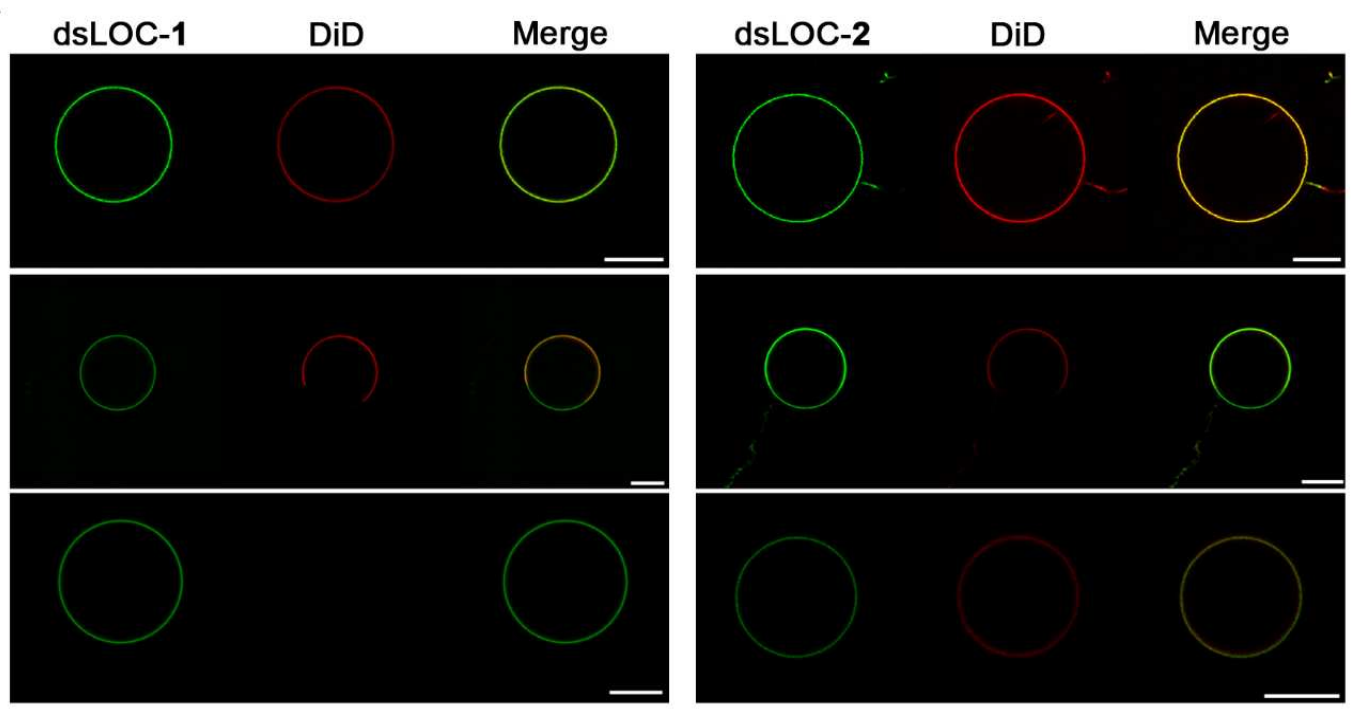

B
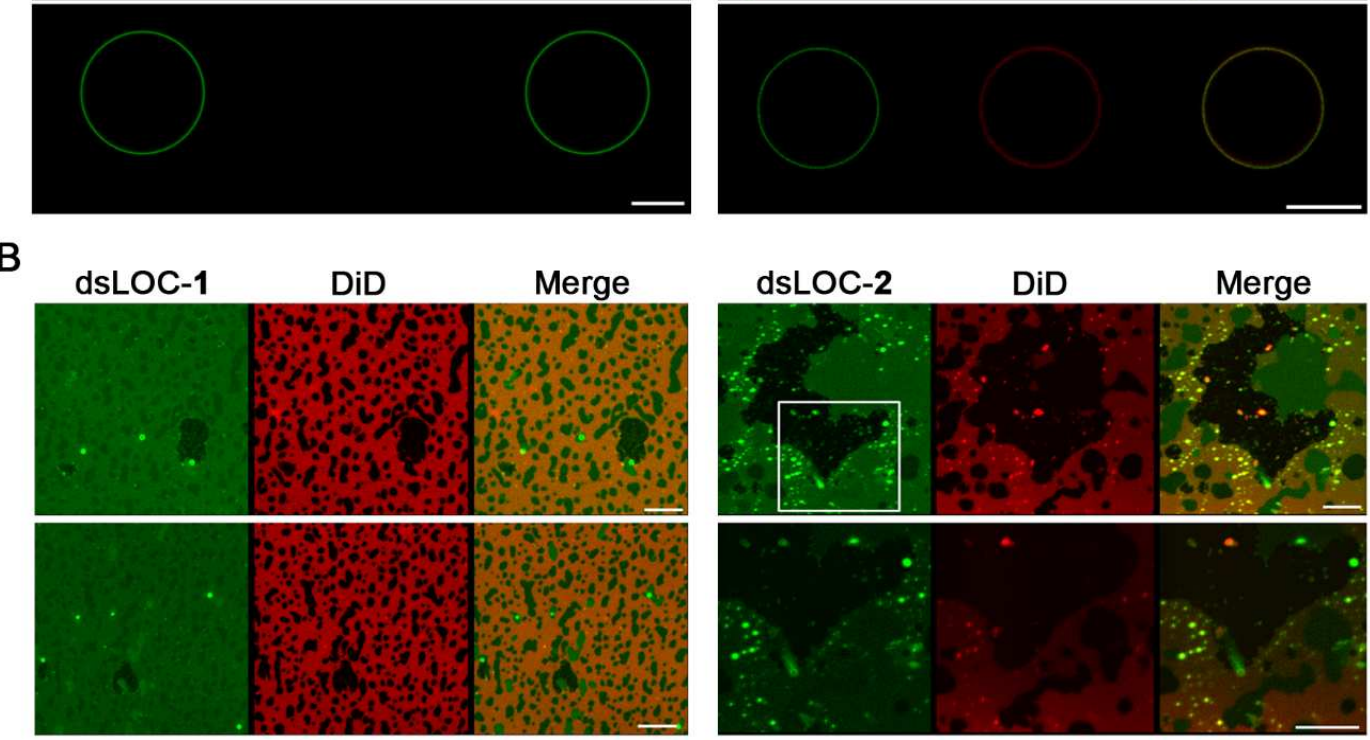
FIGURE 4. Alexa 488-enriched structures formed with dsLOC-1 in DiD-labelled DOPC/eSM/Chol (2:2:1) SPBs. (A) Imaging of Alexa 488-enriched structures from a single SPB zone. Arrowhead and arrow stand for more and less stable Alexa 488-enriched structures, respectively. (B) Imaging of a single Alexa 488-enriched structure. In both cases different z planes were taken from a single SPB zone. Scale bars, $10 \mu \mathrm{m}$.

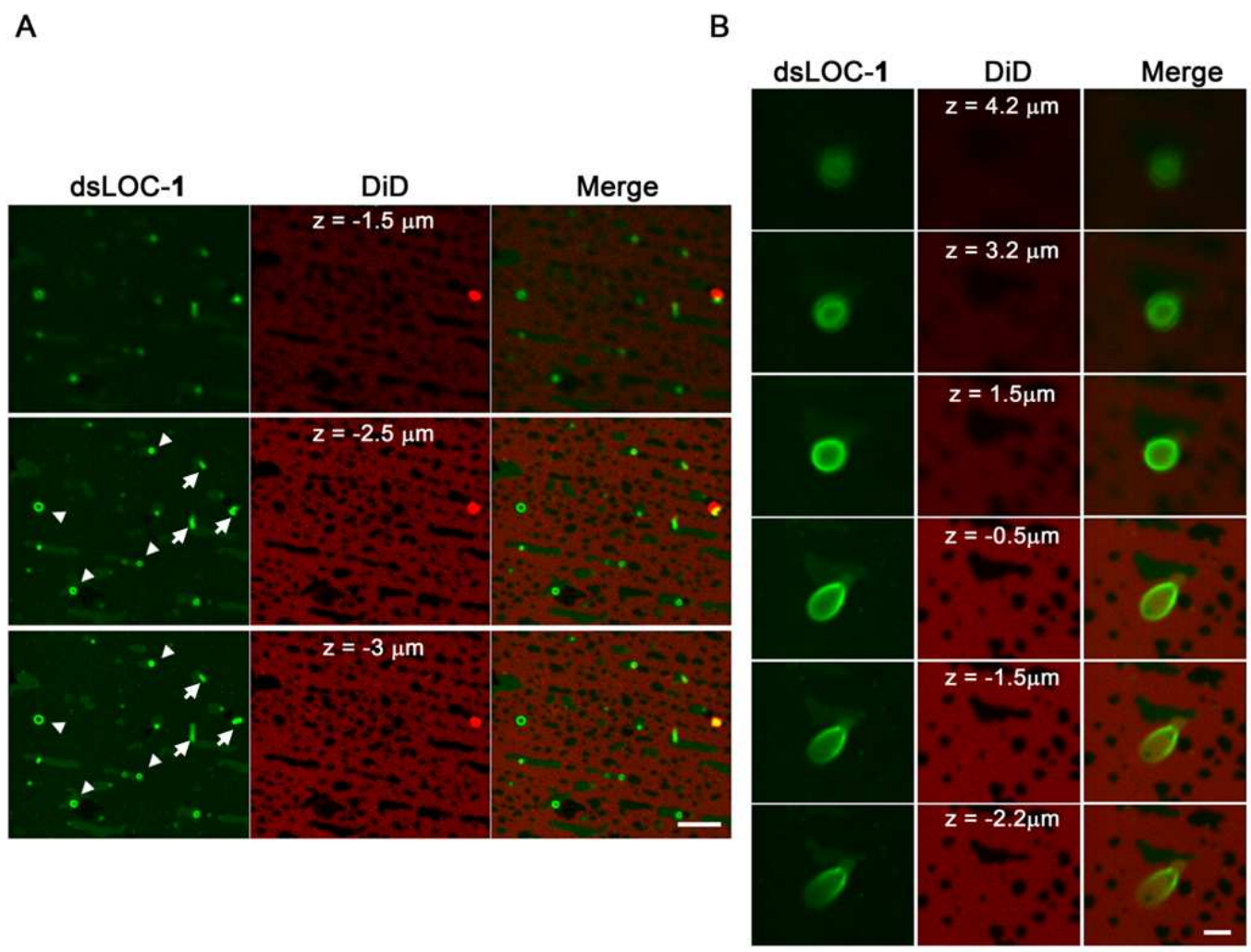


FIGURE 5. LOC-21 internalization pathway in HeLa cells. (A) Representative histograms and (B) flow cytometry values (Geometric Mean of Fluorescence Intensity, GMFI) of the cellular uptake of Alexa 488-labelled LOC-21 in HeLa cells in the presence of endocytic inhibitors. Results are expressed as GMFI of 5 independent experiments performed in duplicate (n.s. for filipin; $\mathrm{p}<0.05$ for CPZ and genistein; $\mathrm{p}<0.01$ for the rest). (C) Representative Western blotting assay of clathrin HC and caveolin-1 protein expression in HeLa cells after treatment with the appropriate siRNA molecule. Bottom graph shows the results of scanning densitometry of the exposed film. Data are expressed as arbitrary units of intensity relative to control value (Control) which stands for untreated sample, results are the mean \pm SD of 2 independent experiments. (D) Cellular uptake of Alexa 488labelled LOC-21 in HeLa cells pretreated with clathrin HC and caveolin-1 siRNAs. Clathrin HC siRNA and caveolin-1 siRNA stand for HeLa cells pretreated with clathrin HC siRNA and caveolin1 siRNA, respectively. Results are expressed as GMFI of 3 independent experiments performed in duplicate ( $\mathrm{p}<0.05$ for clathrin HC siRNA; $\mathrm{p}<0.01$ for caveolin-1 siRNA). 
A

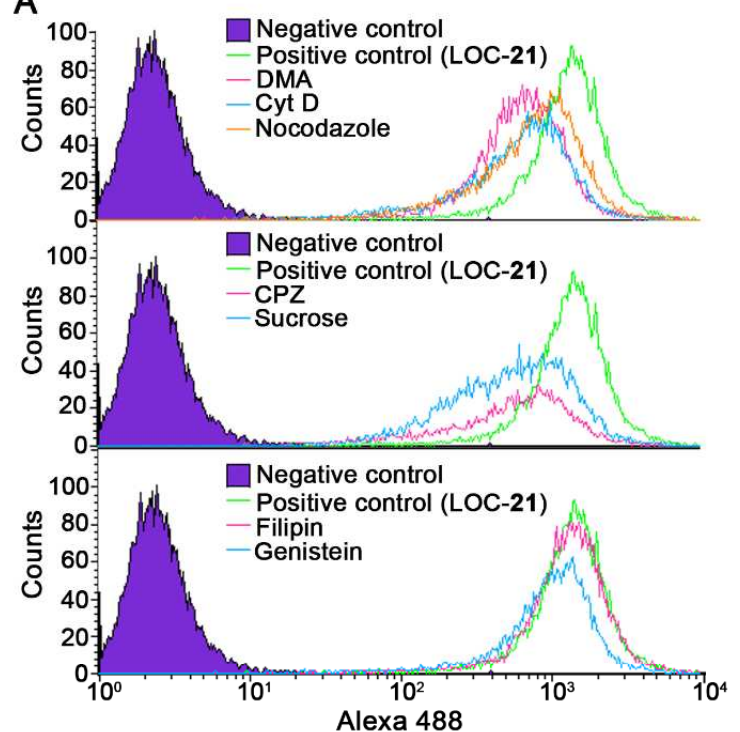

B

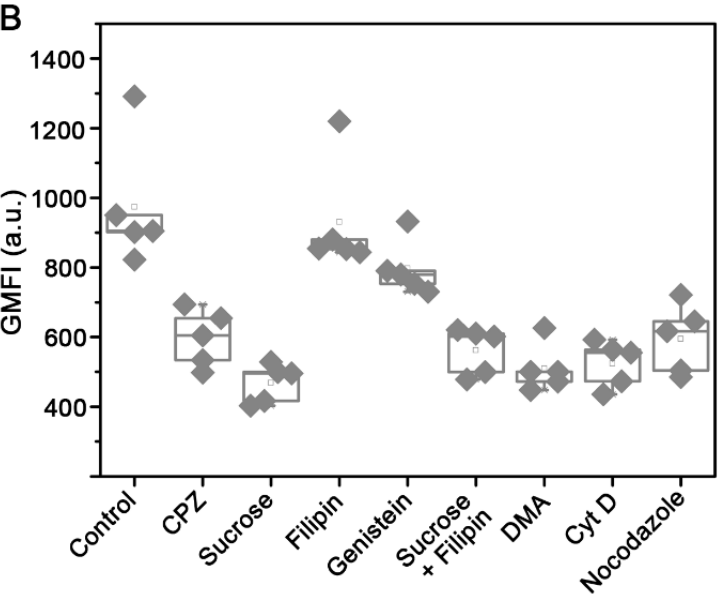

C

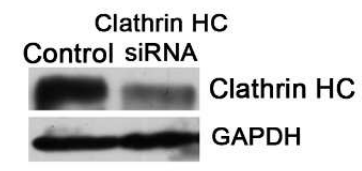

Caveolin-1

Control siRNA
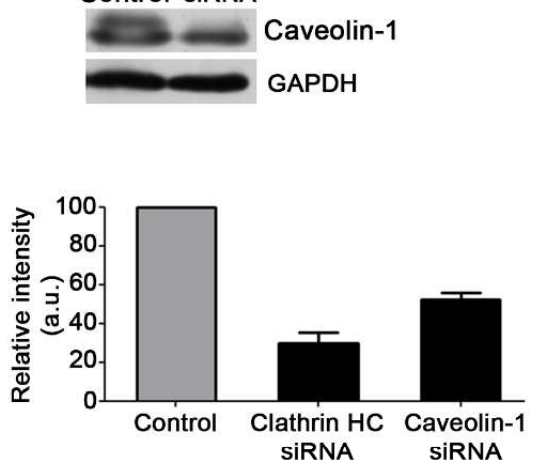

D

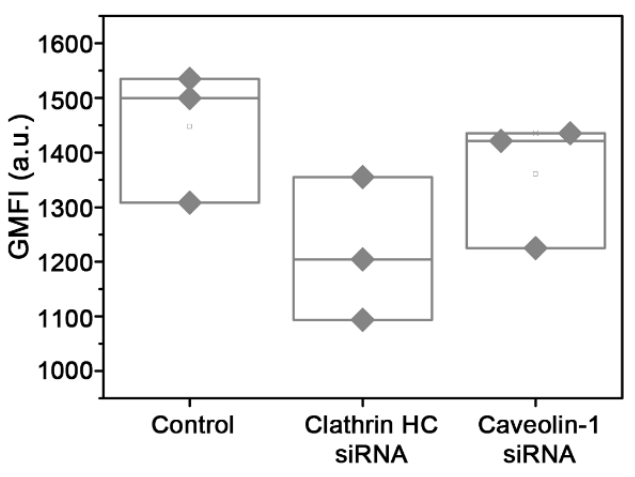


FIGURE 6. Visualization of LOC-21 internalization in HeLa cells with endocytic markers in the presence of endocytic inhibitors. Cells pre-treated with DMA (A), sucrose (B) or filipin (C) were incubated with LOC-21 (green) prior to fixation. (A) Alexa Fluor 546-Phalloidin was used for actin staining (red). (B) Primary antibody to Clathrin HC and Alexa Fluor 633 secondary antibody were used for clathrin staining (magenta). (C) Primary antibody to M6PR and Alexa Fluor 546 secondary antibody were used for late endosome staining (red). In all cases Hoechst 33258 was used for nucleus staining (blue). White boxes stand for zoomed areas.

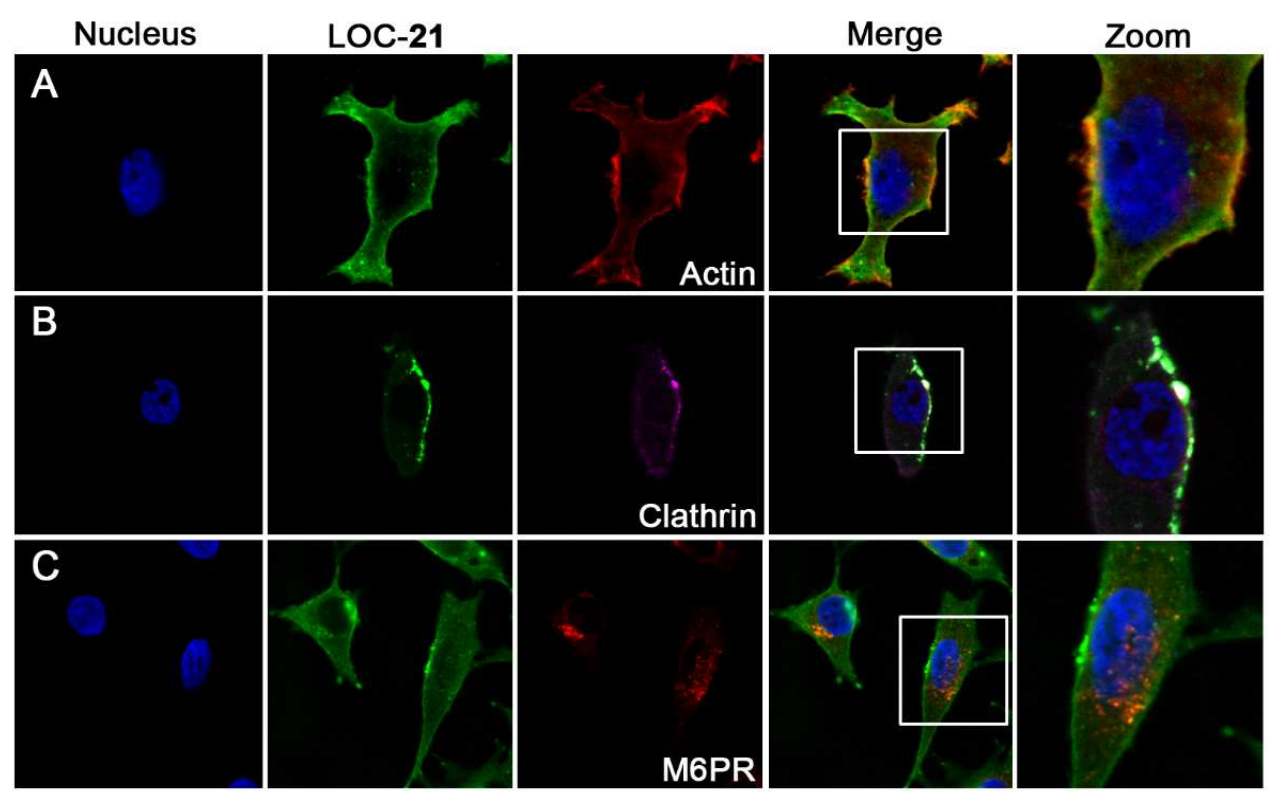

